\title{
Y Dwarf Trigonometric Parallaxes from the Spitzer Space Telescope
}

\author{
Emily C. Martin ${ }^{1,2}$ (1) J. Davy Kirkpatrick ${ }^{2}$ (10), Charles A. Beichman ${ }^{2,3}$, Richard L. Smart ${ }^{4}$, Jacqueline K. Faherty ${ }^{5}$, \\ Christopher R. Gelino $^{2,3}$, Michael C. Cushing ${ }^{6}$ (D), Adam C. Schneider ${ }^{7}$ (D), Edward L. Wright ${ }^{1}$ (D), Patrick Lowrance ${ }^{2}$ (D), \\ James Ingalls $^{2}$ (D) C. G. Tinney ${ }^{8,9}$ (D) , Ian S. McLean ${ }^{1}$, Sarah E. Logsdon ${ }^{1,10}$ (D), and Jérémy Lebreton $^{2}$ (D) \\ ${ }^{1}$ Department of Physics and Astronomy, University of California Los Angeles, 430 Portola Plaza, Box 951547, Los Angeles, CA 90095-1547, USA \\ emartin@astro.ucla.edu \\ 2 IPAC, MS 100-22, Caltech, 1200 East California Blvd., Pasadena, CA 91125, USA \\ ${ }_{4}^{3}$ NASA Exoplanet Science Institute, California Institute of Technology, 770 S. Wilson Ave., Pasadena, CA 91125, USA \\ ${ }^{4}$ Istituto Nazionale di Astrofisica, Osservatorio Astrofisico di Torino, Strada Osservatorio 20, I-10025 Pino Torinese, Italy \\ 5 Department of Astrophysics, American Museum of Natural History, New York, NY 10023, USA \\ ${ }^{6}$ Department of Physics and Astronomy, The University of Toledo, 2801 West Bancroft St., Toledo, OH 43606, USA \\ ${ }_{8}^{7}$ School of Earth and Space Exploration, Arizona State University, Tempe, AZ 85282, USA \\ ${ }^{8}$ Exoplanetary Science at UNSW, School of Physics, UNSW Sydney, NSW 2052, Australia \\ ${ }^{9}$ Australian Center for Astrobiology, UNSW Australia, NSW 2052, Australia \\ ${ }^{10}$ NASA Goddard Space Flight Center, 8800 Greenbelt Rd., Greenbelt, MD 20771, USA \\ Received 2018 April 17; revised 2018 September 5; accepted 2018 September 6; published 2018 November 5
}

\begin{abstract}
Y dwarfs provide a unique opportunity to study free-floating objects with masses $<30 M_{\text {Jup }}$ and atmospheric temperatures approaching those of known Jupiter-like exoplanets. Obtaining distances to these objects is an essential step toward characterizing their absolute physical properties. Using Spitzer's Infrared Array Camera (IRAC) [4.5] images taken over baselines of 2-7 years, we measure astrometric distances for 22 late-T and early Y dwarfs, including updated parallaxes for 18 objects and new parallax measurements for 4 objects. These parallaxes will make it possible to explore the physical parameter space occupied by the coldest brown dwarfs. We also present the discovery of six new late-T dwarfs, updated spectra of two T dwarfs, and the reclassification of a new Y dwarf, WISE J033605.04-014351.0, based on Keck/NIRSPEC J-band spectroscopy. Assuming that effective temperatures are inversely proportional to absolute magnitude, we examine trends in the evolution of the spectral energy distributions of brown dwarfs with decreasing effective temperature. Surprisingly, the Y dwarf class encompasses a large range in absolute magnitude in the near- to mid-infrared photometric bandpasses, demonstrating a larger range of effective temperatures than previously assumed. This sample will be ideal for obtaining mid-infrared spectra with the James Webb Space Telescope because their known distances will make it easier to measure absolute physical properties.
\end{abstract}

Key words: parallaxes - brown dwarfs - stars: individual (WISE J033605.04-014351.0)

Supporting material: figure set, machine-readable tables, tar.gz file

\section{Introduction}

Y dwarfs (Cushing et al. 2011; Kirkpatrick et al. 2012) have effective temperatures $\left(T_{\text {eff }}\right) \lesssim 500 \mathrm{~K}$, are extremely faint, and emit the majority of their light in the mid-infrared. The all-sky, space-based Wide-field Infrared Survey Explorer mission (WISE; Wright et al. 2010) has specifically designed $W 1$ and $W 2$ filter bandpasses such that the $W 1$ filter covers the strong, fundamental $\mathrm{CH}_{4}$ bandhead at $3.3 \mu \mathrm{m}$, a known absorber in the atmospheres of cold brown dwarfs, and the $W 2$ filter centers on the peak of emission expected at $4.5 \mu \mathrm{m}$. Thus, cold brown dwarfs have very red $W 1-W 2$ colors and can be easily identified.

The first $\mathrm{Y}$ dwarfs were confirmed using a combination of ground-based and space-based spectroscopy. With typical $J$ - and $H$-band magnitudes $\gtrsim 19$, these observations are at the limit of the capabilities of the largest ground-based telescopes, and supplemental Hubble Space Telescope (HST) observations are often required. However, the faintest Y dwarf candidates, with near-infrared magnitudes $\gtrsim 23$, are difficult even for $H S T$, and will require James Webb Space Telescope (JWST) observations to fully characterize their atmospheres. Observations of the brightest $\mathrm{Y}$ dwarfs revealed nearly equal flux, sharp emission peaks (in units of $f_{\lambda}$ ) in the shorter wavelength nearinfrared $Y, J$, and $H$ bands, and relatively shallower, broader
$K$-band fluxes (Cushing et al. 2011; Leggett et al. 2016). $\mathrm{CH}_{4}$ and $\mathrm{H}_{2} \mathrm{O}$ are the major absorbers in the atmospheres of $\mathrm{Y}$ dwarfs, carving out large swaths of their spectra in the near- and mid-infrared. Initial atmospheric models (Burrows et al. 2003) suggested that $\mathrm{NH}_{3}$ would also be present in the atmospheres of $\mathrm{Y}$ dwarfs. Observers have yet to find direct spectroscopic evidence of this molecule in the near-infrared (Leggett et al. 2013; Schneider et al. 2015); however, Line et al. (2015) and Line et al. (2017) found unambiguous detections of $\mathrm{NH}_{3}$ in cold brown dwarf spectra using advanced atmospheric retrieval techniques. Such difficulties in directly observing $\mathrm{NH}_{3}$ absorption features suggests that nonequilibrium chemistry likely plays an important role in mixing the atmosphere faster than it can achieve chemical equilibrium (Morley et al. 2014).

For such cold substellar objects to exist at the current age of the universe, they must inherently have lower masses on average than the M, L, and T dwarf field populations. Based on predictions from evolutionary models (e.g., Burrows et al. 2001; Saumon \& Marley 2008), Y dwarfs occupy the mass range of $\sim 1-30 M_{\text {Jup }}$. Y dwarfs represent the very bottom of the stellar/substellar main sequence, as well as the lowest-mass end of the field-mass function, and are thus crucial targets for follow-up to better understand star formation at the lowest masses. 
Y dwarfs share similar temperatures, masses, and chemical compositions with gas-giant exoplanets, making them useful testbeds for atmospheric physics of the coldest objects. Atmospheric observations of exoplanets are difficult because of the extreme contrast needed to differentiate the light of the planet from its host star. Single, free-floating brown dwarfs in the field do not suffer from being outshone by a brighter, more massive companion, and thus make excellent laboratories for studying the atmospheres of planetary-mass objects at temperatures ranging from $\sim 200-500 \mathrm{~K}$ (Beichman et al. 2014; Faherty et al. 2016; Skemer et al. 2016).

Additionally, because Y dwarfs are so small and faint, most of the known Y dwarfs are located within the nearest $\lesssim 15$ pc to the Sun. Y dwarfs that are farther than $\sim 20 \mathrm{pc}$ are too faint to be observable with WISE. The farthest known Y dwarf, WD 0806-661B, at $\sim 19 \mathrm{pc}$, was found as a companion to a white dwarf (Luhman et al. 2011), through a common-proper-motion search of the nearest stellar systems. Recent studies (e.g., Smart et al. 2010; Winters et al. 2017) have focused on completing the census of low-mass stars in the solar neighborhood. Kirkpatrick et al. (2012) presented a preliminary volumelimited survey of the coldest $\left(T_{\text {eff }} \lesssim 1000 \mathrm{~K}\right)$ substellar objects within the nearest $8 \mathrm{pc}$, but were only able to place lower limits on the number density of the coldest and lowest-mass brown dwarfs below $600 \mathrm{~K}$. Precise distances of a larger sample of ultracool brown dwarfs will allow us to better characterize the solar neighborhood down to the lowest masses.

Our current understanding of the star formation process lacks empirical data to place bounds on the lowest mass capable of forming from the collapse and turbulent fragmentation of a massive molecular cloud, if such a bound even exists. The so-called minimum Jeans mass has been examined from a theoretical perspective by several groups (see, e.g., Low \& Lynden-Bell 1976; Bate 2005; Padoan et al. 2007 and references therein) and shown to vary from $\sim 3 M_{\text {Jup }}$ to $\sim 10 M_{\text {Jup }}$. Burgasser (2004) used simulations of varying birthrates and mass functions along with evolutionary models from Burrows et al. (1997) and Baraffe et al. (2003) to show the estimated luminosity functions and temperature distributions that could be produced. The local number density of $\mathrm{Y}$ dwarfs is shown to be the most critical constraint in determining the minimum Jeans mass. Furthermore, the relatively small number of low-mass brown dwarfs that are companions to nearby stars can be used to infer that gravitational instability is not likely to produce objects below $\sim 15 M_{\text {Jup }}$ (Zuckerman \& Song 2009).

Recent studies have presented trigonometric parallaxes and proper motions for small samples of nearby brown dwarfs. Several of these objects were discovered to be within 3 pc (WISE 1049-5319AB, Luhman 2013; WISE 0855-0714, Luhman 2014) and have dramatically altered our understanding of the solar neighborhood since these systems were found to be the third and fourth closest systems to the Sun. Previous studies of the parallaxes of late-T and Y dwarfs include Dupuy \& Kraus (2013) and Leggett et al. (2017), who used data from the Spitzer Space Telescope to measure astrometric fits. Beichman et al. (2014) used a combination of Spitzer and ground-based astrometry, and Smart et al. (2017) and Tinney et al. (2014) both utilized ground-based near-infrared observations to measure parallaxes. Luhman \& Esplin (2016) published initial parallaxes for three $\mathrm{Y}$ dwarfs presented in this paper, using a subset of the data from the Spitzer programs reported here. We provide updated parallaxes for these objects using a longer time baseline.

Our Spitzer parallax program (PI: Kirkpatrick) aims to measure distances to all of the nearby late-T and Y dwarfs within $20 \mathrm{pc}$ that are not being covered by ground-based astrometric monitoring. We are astrometrically monitoring 143 objects with Spitzer/Infrared Array Camera (IRAC) channel 2 imaging through 2018 (Cycle 13). In this paper, we present Spitzer photometry for 27 objects, including preliminary parallaxes for 19 Y dwarfs and 3 late-T dwarfs in our Spitzer parallax program. The Spitzer observations cover baselines of $\sim 2-7$ years.

We also present spectroscopic confirmation and spectrophotometric distance estimates for several AllWISE late-T and Y dwarf candidates with Keck/NIRSPEC J-band observations. The AllWISE processing of the WISE database combined all of the photometry from the original WISE mission and selected high-proper motion candidates (see Kirkpatrick et al. 2014 for the initial results from the AllWISE motion survey). The new brown dwarfs presented in this paper were found in the AllWISE processing but were only recently followed up spectroscopically to confirm their substellar nature.

In Section 2 we present our sample of targets and candidate selection methods. Section 3 describes our ground-based photometric and spectroscopic follow-up. Our Spitzer photometric and astrometric data acquisition and reduction methods are explained in Section 4, and astrometric analysis is detailed in Section 5. We present our results in Section 6, followed by a discussion in Section 7. We summarize our findings in Section 8.

\section{Sample}

Objects in this paper were selected from two separate lists. The first was a list of 19 previously published Y dwarfs (Cushing et al. 2011; Kirkpatrick et al. 2012, 2014; Tinney et al. 2012; Schneider et al. 2015), which includes one object, WISE J033605.04-014351.0 (hereafter WISE 0336-0143), ${ }^{11}$ published earlier as a late-T dwarf (Mace et al. 2013a) but now identified here as an early Y (See Section 3.3). The second was a list of eight objects selected from either the WISE All-Sky Source Catalog or the AllWISE Source Catalog as having colors and magnitudes suggesting a late spectral type $(\geqslant \mathrm{T} 6)$. Specifically, these eight objects - all classified as late-T dwarfs and listed in Table 1 -were selected as (1) having $W 1-W 2>$ $2.7 \mathrm{mag}$ and $W 2-W 3<3.5 \mathrm{mag}$, (2) detected with a signal to noise ratio $(\mathrm{S} / \mathrm{N})>3$ in $W 2$, and (3) not flagged as a known artifact in $W 2$.

\section{Photometric and Spectroscopic Follow-up}

In this paper, we present Spitzer/IRAC channel $1(3.6 \mu \mathrm{m}$ band; hereafter, [3.6]) and channel $2(4.5 \mu \mathrm{m}$ band; hereafter, [4.5]) photometry for all 27 objects. Five of these targets were color-selected too late to have sufficient astrometric monitoring, however we were able to confirm their late-T dwarf nature. We present updated (18) and new (4) parallaxes for the remaining 22 late- $\mathrm{T}$ and $\mathrm{Y}$ dwarfs. Of the $\mathrm{T}$ dwarfs in the sample, if the resulting Spitzer [3.6]-[4.5] color hinted at its substellar nature, it was selected for ground-based near-infrared photometric follow-up. Then, if the $J-W 2$ or $H-W 2$ color further verified the late type, the object was scheduled for

\footnotetext{
11 Source designations are abbreviated as WISE hhmm \pm ddmm. Full designations are given in Table 1.
} 
Table 1

Coordinates, Spectral Types, and Photometry of Target Objects

\begin{tabular}{|c|c|c|c|c|c|c|c|c|c|}
\hline $\begin{array}{l}\text { WISEA } \\
\text { Designation } \\
\text { (1) }\end{array}$ & $\begin{array}{c}\text { Infrared } \\
\text { Sp. Type } \\
\text { (2) }\end{array}$ & References & $\begin{array}{c}J_{\mathrm{MKO}} \\
(\mathrm{mag}) \\
(4)\end{array}$ & $\begin{array}{c}H_{\mathrm{MKO}} \\
(\mathrm{mag}) \\
(5)\end{array}$ & $\begin{array}{c}\text { References } \\
\text { (6) }\end{array}$ & $\begin{array}{c}W 1 \\
(\mathrm{mag}) \\
(7)\end{array}$ & $\begin{array}{c}W 2 \\
(\mathrm{mag}) \\
(8)\end{array}$ & $\begin{array}{c}3.6] \\
(\mathrm{mag}) \\
(9)\end{array}$ & $\begin{array}{c}4.5] \\
(\mathrm{mag}) \\
(10)\end{array}$ \\
\hline J014656.66+423409.9AB & $\mathrm{T} 9+\mathrm{Y}^{\mathrm{a}}$ & 2 & $20.69 \pm 0.07$ & $21.30 \pm 0.12$ & 8 & $>19.137$ & $15.083 \pm 0.065$ & $17.360 \pm 0.089$ & $15.069 \pm 0.022$ \\
\hline J033605.04-014351.0 & $\mathrm{Y} 0^{\mathrm{b}}$ & 1 & $>21.1$ & $>20.2$ & 1 & $18.449 \pm 0.470$ & $14.557 \pm 0.057$ & $17.199 \pm 0.076$ & $14.629 \pm 0.019$ \\
\hline J035000.31-565830.5 & Y1 & 2 & $22.178 \pm 0.073$ & $22.263 \pm 0.135$ & 5 & $>18.699$ & $14.745 \pm 0.044$ & $17.832 \pm 0.131$ & $14.712 \pm 0.019$ \\
\hline J035934.07-540154.8 & Y0 & 2 & $21.566 \pm 0.046$ & $22.028 \pm 0.112$ & 5 & $>19.031$ & $15.384 \pm 0.054$ & $17.565 \pm 0.108$ & $15.357 \pm 0.023$ \\
\hline J041022.75+150247.9 & Y0 & 3 & $19.325 \pm 0.024$ & $19.897 \pm 0.038$ & 5 & $>18.170$ & $14.113 \pm 0.047$ & $16.578 \pm 0.047$ & $14.149 \pm 0.018$ \\
\hline J053516.87-750024.6 & $\geqslant \mathrm{Y} 1:$ & 2 & $22.132 \pm 0.071$ & $23.34 \pm 0.34$ & 5,9 & $17.940 \pm 0.143$ & $14.904 \pm 0.047$ & $17.648 \pm 0.112$ & $15.116 \pm 0.022$ \\
\hline J055047.86-195051.4 & T6.5 & 1 & $17.925 \pm 0.021$ & $\cdots$ & 1 & $18.727 \pm 0.437$ & $15.594 \pm 0.095$ & $16.536 \pm 0.039$ & $15.303 \pm 0.021$ \\
\hline J061557.21+152626.1 & T8.5 & 1 & $18.945 \pm 0.052$ & $\cdots$ & 1 & $>18.454$ & $15.324 \pm 0.117$ & $17.189 \pm 0.057$ & $15.199 \pm 0.019$ \\
\hline J064223.48+042343.1 & $\mathrm{T} 8$ & 1 & $17.677 \pm 0.012$ & $\cdots$ & 1 & $>18.583$ & $15.418 \pm 0.110$ & $16.654 \pm 0.039$ & $15.177 \pm 0.019$ \\
\hline J064723.24-623235.4 & Y1 & 4 & $22.854 \pm 0.066$ & $23.306 \pm 0.166$ & 5 & $>19.539$ & $15.224 \pm 0.051$ & $17.825 \pm 0.128$ & $15.151 \pm 0.021$ \\
\hline J071322.55-291752.0 & Y0 & 2 & $19.98 \pm 0.05$ & $20.19 \pm 0.08$ & 10 & $>18.776$ & $14.462 \pm 0.052$ & $16.646 \pm 0.052$ & $14.208 \pm 0.018$ \\
\hline J073444.03-715743.8 & Y0 & 2 & $20.354 \pm 0.029$ & $21.069 \pm 0.071$ & 5 & $18.749 \pm 0.281$ & $15.189 \pm 0.050$ & $17.605 \pm 0.100$ & $15.271 \pm 0.022$ \\
\hline $\mathrm{J} 082507.37+280548.2$ & Y0.5 & 5 & $22.401 \pm 0.050$ & $22.965 \pm 0.139$ & 5 & $>18.444$ & $14.578 \pm 0.060$ & $17.424 \pm 0.097$ & $14.642 \pm 0.019$ \\
\hline J105130.02-213859.9 & T8.5 & 1 & $18.939 \pm 0.099$ & $19.190 \pm 0.391$ & 11 & $17.301 \pm 0.141$ & $14.596 \pm 0.056$ & $16.467 \pm 0.042$ & $14.640 \pm 0.019$ \\
\hline J105553.62-165216.5 & T9.5 & 1 & $20.703 \pm 0.212$ & $>20.1$ & 1 & $>18.103$ & $15.067 \pm 0.078$ & $17.352 \pm 0.085$ & $15.011 \pm 0.021$ \\
\hline $\mathrm{J} 120604.25+840110.5$ & Y0 & 5 & $20.472 \pm 0.030$ & $21.061 \pm 0.062$ & 5 & $>18.734$ & $15.058 \pm 0.054$ & $17.258 \pm 0.088$ & $15.320 \pm 0.022$ \\
\hline J122036.38+540717.3 & T9.5 & 1 & $20.452 \pm 0.100$ & & 1 & $19.227 \pm 0.517$ & $15.757 \pm 0.091$ & $17.896 \pm 0.101$ & $15.694 \pm 0.022$ \\
\hline J131833.96-175826.3 & $\mathrm{T} 8$ & 1 & $18.433 \pm 0.187^{\mathrm{c}}$ & $17.714 \pm 0.232^{\mathrm{c}}$ & 10 & $17.513 \pm 0.160$ & $14.666 \pm 0.058$ & $16.789 \pm 0.056$ & $14.712 \pm 0.019$ \\
\hline $\mathrm{J} 140518.32+553421.3$ & Y0 pec? & 3 & $21.061 \pm 0.035$ & $21.501 \pm 0.073$ & 5 & $18.765 \pm 0.396$ & $14.097 \pm 0.037$ & $16.850 \pm 0.059$ & $14.069 \pm 0.017$ \\
\hline $\mathrm{J} 154151.65-225024.9^{\mathrm{d}}$ & Y1 & 5 & $21.631 \pm 0.064$ & $22.085 \pm 0.170$ & 5 & $16.736 \pm 0.165$ & $14.246 \pm 0.063$ & $16.512 \pm 0.046$ & $14.227 \pm 0.018$ \\
\hline J163940.84-684739.4 & Y0 pec & 6 & $20.626 \pm 0.023$ & $20.746 \pm 0.029$ & 5 & $17.266 \pm 0.187$ & $13.544 \pm 0.059$ & $16.293 \pm 0.029$ & $13.679 \pm 0.016$ \\
\hline J173835.52+273258.8 & Y0 & 3 & $19.546 \pm 0.023$ & $20.246 \pm 0.031$ & 5 & $17.710 \pm 0.157$ & $14.497 \pm 0.043$ & $16.973 \pm 0.064$ & $14.475 \pm 0.018$ \\
\hline J182831.08+265037.6 & $\geqslant \mathrm{Y} 2$ & 3 & $23.48 \pm 0.23$ & $22.85 \pm 0.24$ & 2,9 & $>18.248$ & $14.353 \pm 0.045$ & $16.907 \pm 0.018$ & $14.321 \pm 0.018$ \\
\hline J205628.88+145953.6 & Y0 & 3 & $19.129 \pm 0.022$ & $19.643 \pm 0.026$ & 5 & $16.480 \pm 0.075$ & $13.839 \pm 0.037$ & $16.068 \pm 0.032$ & $13.905 \pm 0.017$ \\
\hline J220304.18+461923.4 & T8 & 1 & $18.573 \pm 0.017$ & & 1 & $>18.919$ & $14.967 \pm 0.069$ & $16.351 \pm 0.021$ & $14.643 \pm 0.016$ \\
\hline J220905.75+271143.6 & Y0: & 7 & $22.859 \pm 0.128$ & $22.389 \pm 0.152$ & 5 & $>18.831$ & $14.770 \pm 0.055$ & $17.733 \pm 0.121$ & $14.735 \pm 0.019$ \\
\hline J222055.34-362817.5 & Y0 & 2 & $20.447 \pm 0.025$ & $20.858 \pm 0.035$ & 5 & $>18.772$ & $14.714 \pm 0.056$ & $17.180 \pm 0.072$ & $14.742 \pm 0.020$ \\
\hline
\end{tabular}

Notes.

${ }^{a}$ Object is a known binary, so the combined-light magnitudes are not used elsewhere in this paper.

${ }^{\mathrm{b}}$ See Section 3.3 for a discussion on the spectral type of this object.

${ }^{c}$ Photometry is on the 2MASS system, not on the Mauna Kea Observatories (MKO) system. These values are not used elsewhere in this paper because the two photometric systems are not comparable.

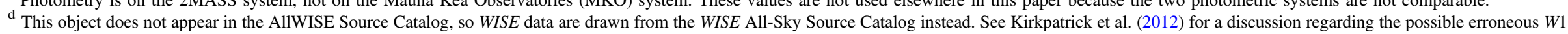
measurement for this object.

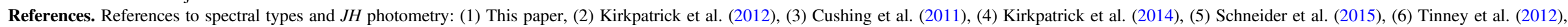

(7) Cushing et al. (2014), (8) Dupuy et al. (2015), (9) Leggett et al. (2013), (10) Leggett et al. (2015), and (11) Mace et al. (2013a).

(This table is available in machine-readable form.) 
Keck/NIRSPEC spectroscopic follow-up. See Figures 1, 7, 8, and 11 of Kirkpatrick et al. (2011) for color trends as a function of spectral type for $\mathrm{T}$ and $\mathrm{Y}$ dwarfs.

\subsection{Ground-based Photometry with Palomar/Wide-Field Infrared Camera (WIRC)}

Near-infrared images of WISE 0336-0143, WISE 0550 -1950 , WISE $0615+1526$, WISE 0642+0423, WISE 1055 -1652 , WISE $1220+5407$, and WISE $2203+4619$ were obtained using the WIRC (Wilson et al. 2003) on the 200 inch Hale Telescope at Palomar Observatory on 2012 January 4 (WISE 0336-0143, WISE 1055-1652), 2014 March 7 (WISE $2203+4619$ ) and 2016 February 26 (WISE 0550-1950, WISE $0615+1526$, WISE $0642+0423$, WISE $1220+5407$ ). WIRC has a pixel scale of 0 "' $2487 /$ pixel providing a total field of view of 8 !7. For each object, fifteen 2-minute images were obtained in the $J$ filter (30 minutes total exposure time). The sky was clear during the observations on all nights.

Images obtained in 2012 and 2014 were reduced using a suite of IRAF scripts and FORTRAN programs provided by T. Jarrett. These scripts first linearize and dark subtract the images. From the list of input images, a sky frame and flat field image are created and subtracted from and divided into (respectively) each input image. At this stage, WIRC images still contain a significant bias that is not removed by the flat field. Comparison of Two Micron All Sky Survey (2MASS; Skrutskie et al. 2006) and WIRC photometric differences across the array shows that this flux bias has a level of $\approx 10 \%$ and the pattern is roughly the same for all filters. Using these 2MASS-WIRC differences for many fields, we can create a flux bias correction image that can be applied to each of the "reduced" images.

In 2014 April, the primary science-grade detector experienced a catastrophic failure and was replaced with a lower quality engineering-grade detector (there are more cosmetic defects, for example). The previous reduction scripts were finetuned for the original detector and produced sub-optimal results with the new chip. A WIRC reduction package written in IDL by J. Surace was used for the 2016 data as it was able to better handle the nonuniformity in one of the quadrants. In addition to the quadrant cleaning, the Surace package differed from the Jarrett package in that the reduced data from the former did not exhibit, and thus did not require, a flux bias correction. The other data reduction steps were essentially the same. The processed frames were mosaicked together using a median and had their astrometry and photometry calibrated using 2MASS stars in the field. ${ }^{12}$

Table 1 lists the photometry, using Vega system magnitudes. Additional photometry for the remaining targets in this sample was taken from the literature. The majority of the near-infrared photometry listed in Table 1 is on the MKO system, though some of the Y dwarfs have synthetic photometry measured with $H S T$ and corrected to match MKO filter profiles (see Schneider et al. 2015 for further details). We caution the reader that the photometric filter system can significantly change the near-infrared photometry of Y dwarfs.

\subsection{Ground-based Spectroscopy with Keck/NIRSPEC}

Using the NIRSPEC instrument at the W. M. Keck Observatory (McLean et al. 1998), we made $J$-band spectroscopic

$\overline{12}$ The mosaicked images are available in a tarball online. observations of four targets from the original Spitzer Parallax Program with unknown or uncertain spectral types: WISE 0336 -0143, WISE 1051-2138, WISE 1055-1652, and WISE 1318 -1758 . We observed an additional five targets that were likely late-type $\mathrm{T}$ or $\mathrm{Y}$ dwarfs based on their $W 1-W 2$ colors from the AllWISE processing (Kirkpatrick et al. 2014). Spectral types and observation information for these targets are listed in Table 2. All targets were observed using $\mathrm{AB}$ nod pairs along the 0 ". 57 (3-pixel) slit, producing a spectral resolution of $R=\lambda / \Delta \lambda \sim$ 1500 per resolution element.

Spectroscopic reductions were made using a modified version of the REDSPEC package, ${ }^{13}$ following a similar procedure to Mace et al. (2013a). Frames were spatially and spectrally rectified to remove the instrumental distortion on the image plane of the detector. Frames were then backgroundsubtracted and divided by a flat field. Spectra from each nod pair were extracted by summing over 9-11 pixels before combining the nods. The extracted spectrum was then divided by an A0V calibrator spectrum to remove telluric features and lastly, corrected for barycentric velocity. Observations made of the same target on separate nights were combined into a single spectrum after being reduced separately. Raw spectra in this paper are available in the Keck Observatory $\operatorname{Archive}^{14}$ and reduced spectra are available on the NIRSPEC Brown Dwarf Spectroscopic Survey website. ${ }^{15}$

\subsection{New Late-T and Y Dwarfs and Updated Spectral Types}

Here we present new and updated spectral types for nine objects in our sample that we observed with NIRSPEC. $J$ band spectra for these objects, and the spectral standards used to classify them are shown in Figure 1.

WISE 0336-0143 was originally classified as T8: by Mace et al. (2013a). In 2016, we sought to re-observe WISE 0336 -0143 for two reasons. First, the spectrum published in Mace et al. (2013a) had a low $\mathrm{S} / \mathrm{N}$ and we wished to obtain a higher $\mathrm{S} / \mathrm{N}$ spectrum. Second, we hypothesized based on its [3.6][4.5] color of 2.57 mag that WISE 0336-0143 should be much colder than a T8 to explain its extreme redness. Typical [3.6][4.5] colors for T8 objects are $\sim 1.5-2 \mathrm{mag}$ (see Figure 7 in Mace et al. 2013b; WISE 0336-0143 is the obvious T8 outlier in that plot.) In Figure 2, we plot the normalized NIRSPEC spectra of the 2011 and 2016 observations. The 2016 observations match much better to a $\mathrm{Y}$ dwarf (see also Figure 1), so we will henceforth classify this object as a Y0:. We have only been able to obtain limits on the near-infrared photometry for this object. With $J>21$, WISE 0336-0143 will require additional observations with an 8 or $10 \mathrm{~m}$ class ground-based telescope, or observations with HST or JWST to further characterize its spectrum.

WISE 0550-1950, WISE 0615+1526, WISE 0642+0423, WISE $1220+5407$, and WISE $2203+4619$ are new T dwarfs found using the AllWISE color cuts discussed in Section 2. We find spectral types of T6.5, T8.5, T8, T9.5, and T8, respectively, based on comparison of their $J$-band spectra to spectral standards.

WISE 1051-2138 was given a spectral type of T9: in Mace et al. (2013a). Our re-observed spectrum, shown in Figure 1, indicates that this object should be classified as T8.5.

\footnotetext{
$\overline{13}$ Available at http://www2.keck.hawaii.edu/inst/nirspec/redspec.html.

14 https://koa.ipac.caltech.edu

15 http://bdssarchive.org
} 

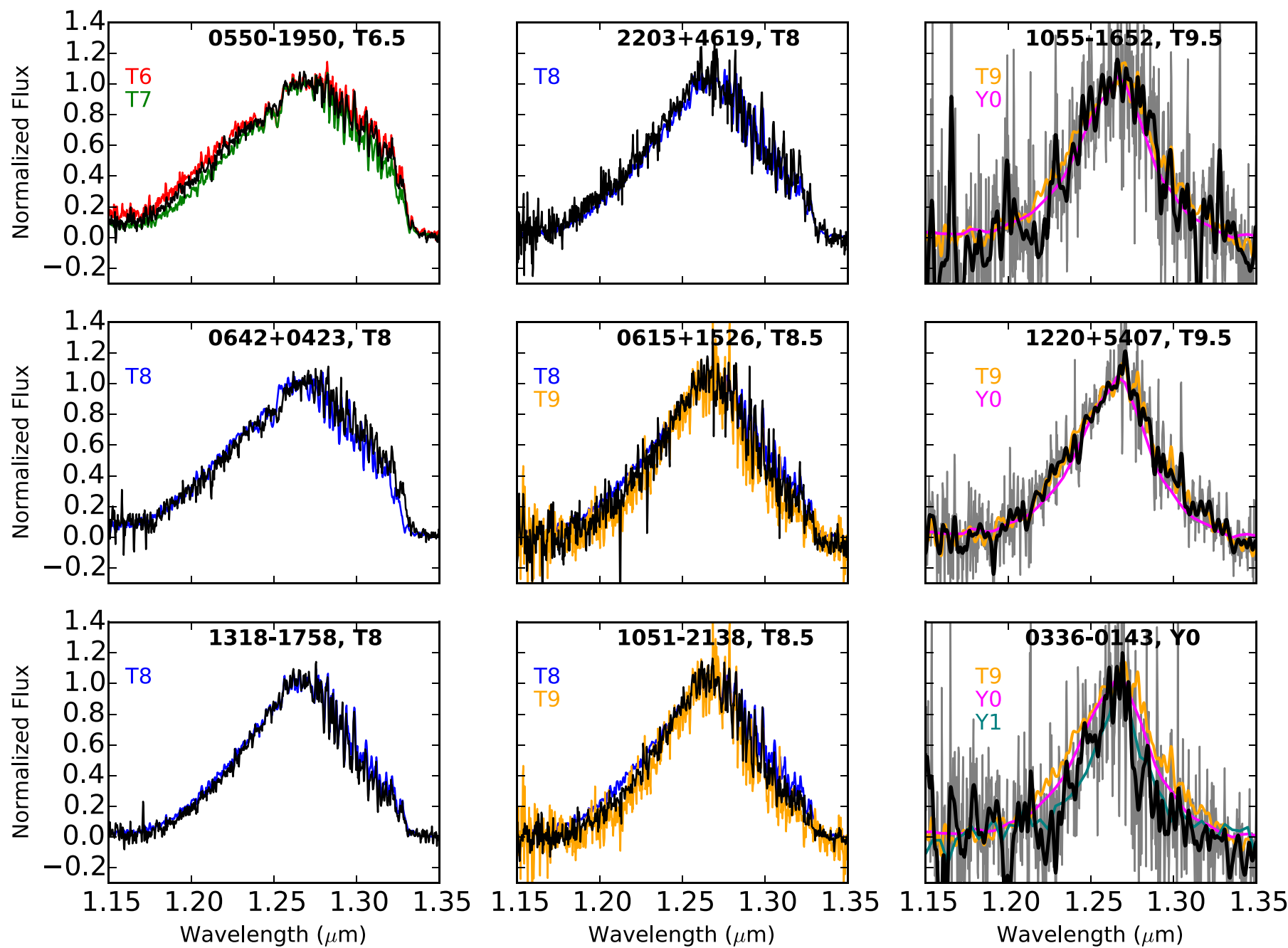

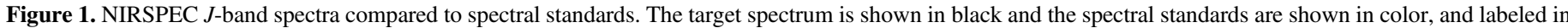

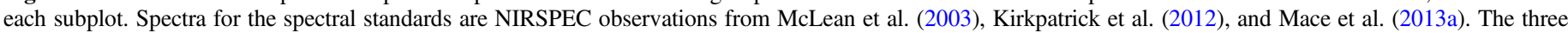

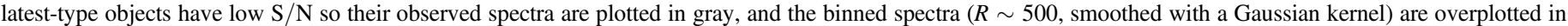
black.

Table 2

NIRSPEC Observations

\begin{tabular}{|c|c|c|c|c|c|}
\hline Short Name & $\mathrm{SpT}$ & UT Date of Observation & Integration Time [s] & A0V Calibrator & Seeing Conditions \\
\hline WISE $0336-0143$ & $\mathrm{Yo}^{\mathrm{a}}$ & 2016 Feb 10 & 2400 & HD 27700 & clear \\
\hline WISE 0550-1950 & T6.5 & 2016 Feb 10 & 3000 & HD 44704 & clear \\
\hline WISE $0615+1526$ & $\mathrm{~T} 8.5$ & 2016 Feb 01 & 1200 & HD 43583 & clear \\
\hline$\ldots$ & $\ldots$ & 2016 Feb 11 & 3000 & HD 43583 & clear \\
\hline WISE $0642+0423$ & $\mathrm{~T} 8$ & 2016 Feb 01 & 4200 & HD 43583 & clear \\
\hline WISE $1051-2138$ & $\mathrm{~T} 8.5$ & 2016 Feb 11 & 4200 & HD 95642 & clear \\
\hline WISE $1055-1652$ & T9.5: & 2016 Feb 01 & 6600 & HD 98884 & clear \\
\hline$\cdots$ & $\cdots$ & 2016 Feb 10 & 3600 & HD 92079 & clear \\
\hline WISE $1220+5407$ & T9.5 & 2016 Feb 01 & 1800 & $81 \mathrm{UMa}$ & variable seeing \\
\hline$\cdots$ & $\cdots$ & 2016 Feb 11 & 3600 & HD 99966 & clear \\
\hline WISE $1318-1758$ & $\mathrm{~T} 8$ & 2016 Feb 11 & 2400 & HD 112304 & windy \\
\hline WISE 2203+4619 & $\mathrm{T} 8$ & 2014 Oct 06 & 4800 & HD 219238 & clear \\
\hline
\end{tabular}

Note.

${ }^{a}$ See Section 3.3 for a discussion on the spectral type of this object.

WISE 1055-1652 was placed on our parallax program without having an observed spectrum to confirm its substellar nature. We present the discovery of this new T9.5: dwarf.
WISE 1318-1758 was classified as a T9: in Mace et al. (2013b) based on a noisy Palomar/TripleSpec spectrum and we re-classify it here as a T8. As shown in Figure 1, 
Table 3

Spitzer Observations

\begin{tabular}{|c|c|c|c|}
\hline $\begin{array}{l}\text { Short Name } \\
\text { (1) }\end{array}$ & $\begin{array}{c}\text { Spitzer Program \# (\# of [4.5] Epochs) } \\
(2)\end{array}$ & $\begin{array}{l}\text { MJD Range of Observations } \\
\text { (3) }\end{array}$ & $\begin{array}{l}\text { AORs for Photometry } \\
\text { (4) }\end{array}$ \\
\hline WISE $0146+4234$ & 70062(1), 80109(2), 90007(12) & $55656.0-56768.1$ & 41808128 \\
\hline WISE 0336-0143 & 70062(1), 80109(1), 90007(12) & $55663.2-56777.7$ & 41462784 \\
\hline WISE $0359-5401$ & 70062(2), 80109(2), 90007(12) & $55457.2-57035.8$ & 40819712 \\
\hline WISE $0410+1502$ & 70062(2), 80109(2), 90007(12) & $55490.0-56792.5$ & 40828160 \\
\hline WISE $0535-7500$ & $70062(2), 80109(1), 90007(10)$ & $55486.2-56875.6$ & 41033472 \\
\hline WISE $0642+0423$ & $11059(1)$ & 57175.1 & 52670208 \\
\hline WISE $0647-6232$ & 70062(2), 80109(1), 90007(10) & $55458.4-56887.1$ & 40829696 \\
\hline WISE 0713-2917 & 80109(1), 90007(12) & $55928.8-56856.5$ & 44568064 \\
\hline WISE $0734-7157$ & 70062(1), 80109(1), 90007(10) & $55670.6-56790.7$ & 41754880 \\
\hline WISE $0825+2805$ & 80109(2), 90007(12) & $55933.9-56849.0$ & 44221184 \\
\hline WISE $1051-2138$ & 70062(1), 90007(11) & $55633.6-56903.4$ & 41464320 \\
\hline WISE $1405+5534$ & 70062(1), 80109(2), 90007(10) & $55583.1-56902.1$ & 40836864 \\
\hline WISE $1541-2250$ & $70062(1), 80109(2), 90007(12)$ & $55664.9-56812.0$ & 41788672 \\
\hline WISE $1639-6847$ & $90007(12), 11059(1)$ & $56431.7-57175.3$ & $52672000^{\mathrm{a}}$ \\
\hline WISE $1738+2732$ & 70062(2), 80109(2), 90007(12) & $55457.5-56864.6$ & 40828416 \\
\hline WISE $1828+2650$ & 551(1), 70062(1), 80109(2), 90007(12) & $55387.3-56878.5$ & $39526656,39526912^{\mathrm{b}}$ \\
\hline WISE $2056+1459$ & 70062(2), 80109(2), 90007(12) & $55540.0-57049.0$ & 40836608 \\
\hline WISE $2203+4619$ & $10135(1)$ & 56922.9 & 50033152 \\
\hline WISE $2209+2711$ & $70062(2), 80109(1), 90007(12)$ & $55561.9-56925.3$ & 40821248 \\
\hline WISE $2220-3628$ & 80109(2), 90007(12) & $55949.1-56902.9$ & 44552448 \\
\hline
\end{tabular}

Notes.

${ }^{a}$ This high motion object was blended with a background star during our original observation in program 80109. We reacquired this observation during program 11059 to make up for the loss of a [4.5] astrometric epoch and the loss of our sole [3.6] photometric data point.

$\mathrm{b}$ The [3.6] and [4.5] observations of this object in program 551 were broken into separate AORs but were observed concurrently.

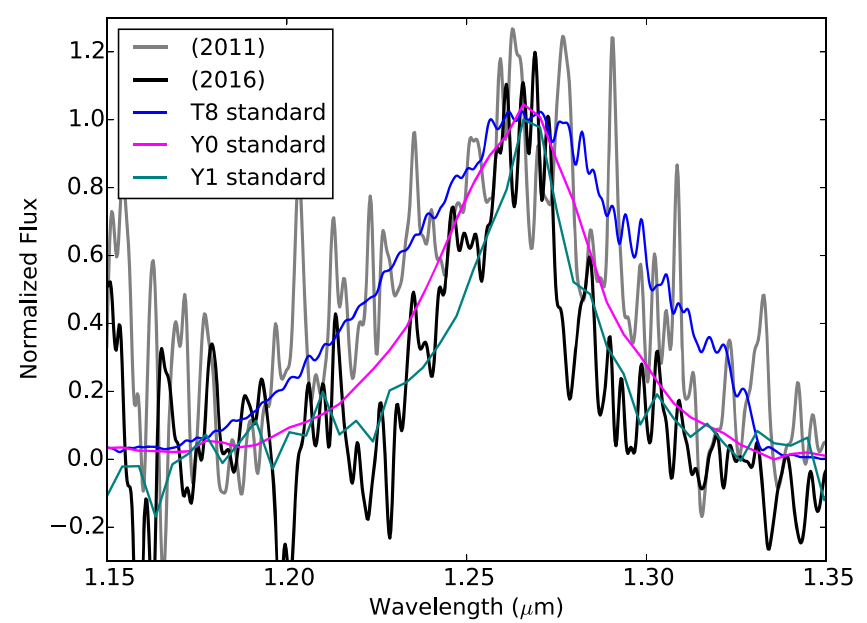

Figure 2. Comparison of the (smoothed) NIRSPEC spectra of WISE 0336 -0143 as observed in 2011 by Mace et al. (2013a) (gray) and as observed in 2016 (this paper, black). The T8 (blue), Y0 (magenta), and Y1 (teal) spectral standards are overplotted for comparison.

the T8 spectral standard is a very good match for WISE $1318-1758$.

\section{Spitzer Astrometric Followup}

In order to measure distances for these ultracool dwarfs, we undertook an astrometric campaign using Spitzer IRAC [4.5] images spanning baselines of $\sim 2-7$ years. We have utilized data from six Spitzer programs (Table 3) in our analysis. Of these, program 90007 was specifically designed for parallax and proper motion measurements.

\subsection{Observations}

Spitzer IRAC [4.5] images have a field of view of 5.2 on a side, over $256 \times 256$ pixels, producing a pixel scale of 1 ." $2 \mathrm{pix}^{-1}$. The full-width at half-maximum for a centered point response function (PRF) is 1."8, for the warm mission. The raw images have a maximum optical distortion of 1.6 pixels, on the edge of the array. During Spitzer cryogenic operations, [3.6] was more sensitive than [4.5]. After cryogen depletion, however, the deep image noise ${ }^{16}$ was found to be $12 \%$ worse in [3.6] and $10 \%$ better in [4.5], making the channels more comparable in sensitivity for average field stars ([3.6]-[4.5] 0 mag) during warm operations (Carey et al. 2010). The behavior of latent images from bright objects was also found to change during warm operations; whereas latents in [4.5] decay rapidlytypically within 10 minutes-[3.6] latents decay on timescales of hours. Moreover, the [4.5] intrapixel sensitivity variation (also known as the pixel phase effect) is about half that of [3.6]. Given these points, the fact that the PRF is better sampled in [4.5] than in [3.6], and the fact that our cold brown dwarfs are also much

\footnotetext{
${ }^{16}$ See "Warm IRAC Characteristics" at http://irsa.ipac.caltech.edu/data/ SPITZER/docs/irac/ for a summary of each of the effects discussed here.
} 
brighter in [4.5] than in [3.6] $(1.0<[3.6]-[4.5]<3.0 \mathrm{mag}$; Figure 11 of Kirkpatrick et al. 2011), we chose to do our imaging in [4.5]. All [4.5] Spitzer/IRAC observations of the targets, the MJD range of usable data for each source, and the number of epochs available in each program, are given in Table 3.

Our primary program, Program 90007, used a total integration time of $270 \mathrm{~s}$ per epoch so that all targets would have $\mathrm{S} / \mathrm{N}>100$ in [4.5]. To smear out the effects of intrapixel sensitivity variation, which can bias the astrometry in a frame, we chose a 9-point random dither pattern with $30 \mathrm{~s}$ exposures per dither. Dither sizes vary for this setup, but are on the order of $\sim 5^{\prime \prime}-30^{\prime \prime} .{ }^{17}$ To keep the number of common reference stars between individual exposures high, we chose a dither pattern of medium scale. Timing constraints were imposed so that there was one sample within a few days of maximum parallax factor with (usually) evenly spaced samples throughout the rest of the target's visibility period.

We also used [4.5] data taken as part of earlier programs 551, 70062, and 80109 as well the later program 11059 (PI: Kirkpatrick) to increase the time baseline to help disentangle proper motion from parallax. Program 11059 used the same observing setup as program 90007, described above. All the other programs from which we utilized data (except 551; PI: Mainzer) used a frame time of $30 \mathrm{~s}$ and a 5-point cycling dither pattern with medium scale, and observations were obtained in both [3.6] and [4.5]. In anticipation of parallax program 90007, we used the same [4.5] setup to re-observe our most promising targets during programs 70062 and 80109 after the original [3.6]+[4.5] Astronomical Observation Request (AOR) was completed.

Program 551, which targeted only WISE $1828+2650$, used a frame time of $100 \mathrm{~s}$ and a 36-point Reuleaux with medium dither in [3.6] and a frame time of $12 \mathrm{~s}$ and a 12-point Reuleaux pattern of medium dither in [4.5]. Program 10135 (PI: Pinfield), which targeted only WISE $2203+4619$, used a frame time of $30 \mathrm{~s}$ and a 16-point spiral dither pattern of medium step in both channels; in this case, two exposures were taken at each dithered position.

\subsection{Astrometric and Photometric Data Reductions}

We used the Spitzer Heritage Archive ${ }^{18}$ to download all of the basic calibrated data (BCD) at [4.5] for the programs listed in Table 3. Data were reduced using the Mosaicker and Point Source Extractor $\left(\mathrm{MOPEX}^{19}\right)$ with customized scripts created following instructions in the MOPEX handbook. These scripts use the individually dithered BCD files to create a coadded image at each epoch (i.e., for each AOR) and to detect and characterize sources on the resulting coadd.

The data and scripts have been modified in two ways to utilize new knowledge gained during the Spitzer warm mission. First, the headers of the BCD files available at the Spitzer Heritage Archive have been updated to include a new Spitzerproduced fifth-order distortion correction for the IRAC camera, which is an improvement over the third-order correction included previously (Lowrance et al. 2014). Second, the PRF

\footnotetext{
17 For more information on dithers, see https://irsa.ipac.caltech.edu/data/ SPITZER/docs/irac/calibrationfiles/dither/.

18 Available at http://irsa.ipac.caltech.edu/.

19 Available at http://irsa.ipac.caltech.edu/data/SPITZER/docs/dataanalysistools/ tools/mopex/.
}

employed by the code is one created specifically for use on Spitzer warm data, ${ }^{20}$ sampled onto a $5 \times 5$ grid to account for small changes in shape across the array. The MOPEX code performs a simultaneous chi-squared minimization ${ }^{21}$ using fits of the PRF to the stack of individual frames to measure the photometry and position of the source in that AOR. It should be noted that the random dithers will help to zero out the astrometric bias caused by the intrapixel distortion in each individual frame (Ingalls et al. 2012), so this effect did not have to be specifically addressed in our reduction methodology.

Our [3.6] observations were run identically to the [4.5] data discussed above. We divided the resulting PRF-fit fluxes by the appropriate [3.6] and [4.5] correction factors (1.021 and 1.012, respectively) indicated in Table C. 1 of the IRAC Instrument Handbook $^{22}$ and converted these fluxes to magnitudes using the [3.6] and [4.5] zero points of $280.9 \pm 4.1 \mathrm{Jy}$ and $179.7 \pm 2.6 \mathrm{Jy}$, respectively, as given in Table 4.1 of the same document. The final [3.6] and [4.5] photometry is listed in columns $9-10$ of Table 1.

Prior studies have shown that the amplitude of [3.6] and/or [4.5] variability in T0-T8 dwarfs can can reach the $10 \%$ level, with some objects varying more in one band than the other (Metchev et al. 2015). This amplitude increases at later spectral types. In fact, one Y dwarf, WISE $1405+5534$, has already been observed to vary at levels as high as $3.5 \%$ in [3.6] and [4.5] based on a limited data set (Cushing et al. 2016). Another Y dwarf observed for variability, WISE 1738 +2732 , showed peak-to-peak variability of $\sim 3 \%$, at [4.5] with potentially up to $30 \%$ variability in the near-infrared (Leggett et al. 2016). Therefore, our tabulated values list photometry only for the one AOR having concurrent [3.6] and [4.5] observations so that the resulting [3.6]-[4.5] value represents a physical snapshot of the color at a specific time rather than a possibly non-physical color created from disparate epochs of [3.6] and [4.5] observations. The AORs from which the [3.6] and [4.5] photometry is measured are listed in Column 4 of Table 3.

The centroid locations determined by the MOPEX routine on each of the epochal coadds (average positions across multiple dithers) were then used as the fundamental source of our astrometric measurements. Our resulting inputs to our astrometric fitting routine at each epoch were the source location, time of observation of the middle frame, and geometric coordinates of Spitzer during the observations.

\section{Astrometric Analysis}

Using the astrometric measurements described in the previous section, we then re-registered each frame onto a common reference frame, determined positional uncertainties, and then solved for the proper motion and parallax of each source, as described below. Target coordinates at each epoch are recorded in Table 4 and our final astrometric solutions are detailed in Table 5.

\footnotetext{
${ }^{20}$ For more information on the PRF maps, see http://irsa.ipac.caltech.edu/ data/SPITZER/docs/irac/calibrationfiles/psfprf/.

21 For more information, see http://irsa.ipac.caltech.edu/data/SPITZER/docs/ dataanalysistools/tools/mopex/mopexusersguide/88/\#_Toc320000081.

${ }^{22}$ Available at http://irsa.ipac.caltech.edu/data/SPITZER/docs/irac/ iracinstrumenthandbook/.
} 

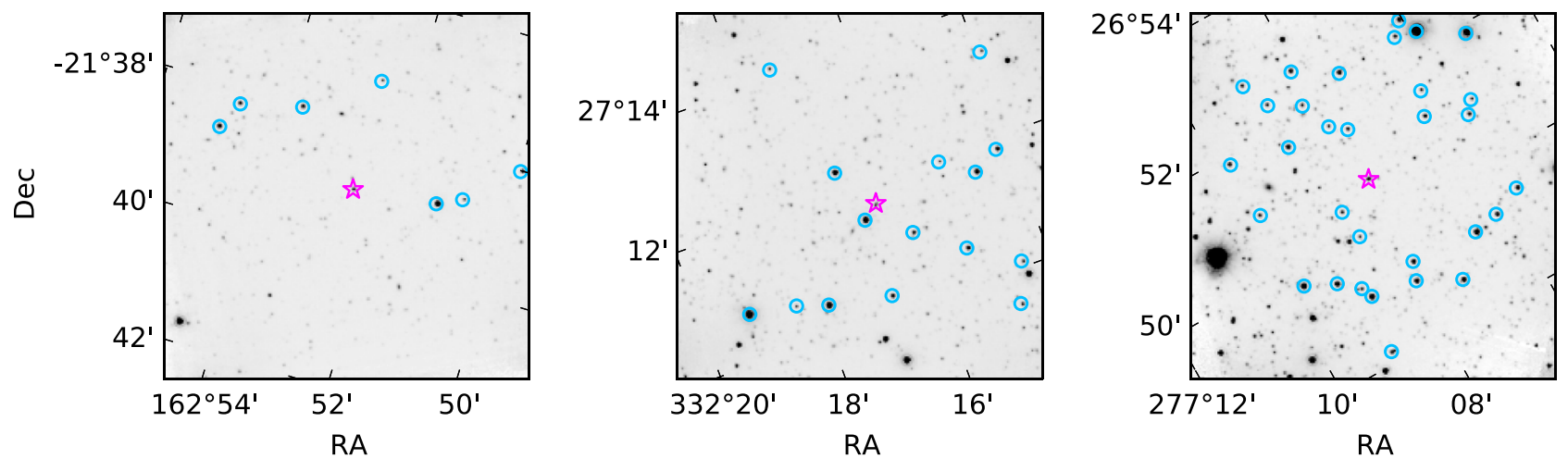

Figure 3. Supermosaic frames of WISE 1051-2138, WISE 2209+2711, and WISE $1828+2650$, from left to right. Reference stars in each of the fields are circled in blue. Targets are marked by a magenta star. These frames show examples of different target fields, ranging from few reference stars to many.

Table 4

Target Coordinates at Each Epoch

\begin{tabular}{|c|c|c|c|c|c|}
\hline $\begin{array}{l}\text { Name } \\
\text { (1) }\end{array}$ & $\begin{array}{l}\text { R.A. } \\
\text { (deg) } \\
(2)\end{array}$ & $\begin{array}{c}\text { decl. } \\
\text { (deg) } \\
(3)\end{array}$ & $\begin{array}{c}\Delta \text { R.A. } \\
(\operatorname{arcsec}) \\
(4)\end{array}$ & $\begin{array}{c}\Delta \text { decl. } \\
(\operatorname{arcsec}) \\
(5)\end{array}$ & $\begin{array}{c}\text { Obs. Date } \\
\text { MJD } \\
(6)\end{array}$ \\
\hline WISE $0146+4234$ & $\begin{array}{l}26.7360341575 \\
26.7358573158 \\
26.7358123414 \\
26.7355032955 \\
26.7355150149 \\
26.7355269728 \\
26.7355337547 \\
26.7356177374 \\
26.735610704 \\
26.7356193002 \\
26.7356217406 \\
26.7356996309 \\
26.7356927974 \\
26.7356863574 \\
26.7356981636\end{array}$ & $\begin{array}{l}42.5694215003 \\
42.5694154093 \\
42.569430009 \\
42.5693971935 \\
42.569396338 \\
42.5694010066 \\
42.5694111367 \\
42.5694257248 \\
42.5694074714 \\
42.5694181532 \\
42.5694100106 \\
42.5694108774 \\
42.5694167189 \\
42.5694080177 \\
42.5694173971\end{array}$ & $\begin{array}{l}0.02 \\
0.02 \\
0.02 \\
0.02 \\
0.02 \\
0.02 \\
0.02 \\
0.02 \\
0.02 \\
0.02 \\
0.02 \\
0.02 \\
0.02 \\
0.02 \\
0.02\end{array}$ & $\begin{array}{l}0.02 \\
0.02 \\
0.02 \\
0.02 \\
0.02 \\
0.02 \\
0.02 \\
0.02 \\
0.02 \\
0.02 \\
0.02 \\
0.02 \\
0.02 \\
0.02 \\
0.02\end{array}$ & $\begin{array}{l}55656.090572 \\
55993.0453281 \\
56215.0761909 \\
56768.0673261 \\
56758.3544525 \\
56750.4605196 \\
56737.3844441 \\
56616.0666252 \\
56602.4785276 \\
56592.4703411 \\
56579.1881263 \\
56393.1344496 \\
56388.816662 \\
56372.3134272 \\
56364.2577053\end{array}$ \\
\hline
\end{tabular}

(This table is available in its entirety in machine-readable form.)

\subsection{Coordinate Reregistration}

Prior to fitting our astrometric solutions for each target, we re-registered the coordinates of our targets in each epoch onto a single reference frame. We chose to align our coordinates to those provided by the Gaia Mission in Data Release 1 (DR1; Gaia Collaboration et al. 2016a, 2016b). These positions are the best that are currently available across the whole sky. 2MASS positions, which provide the basis for the WCS coordinates given by MOPEX/APEX, have positional uncertainties on the order of $\sim 70$ mas (McCallon et al. 2007), while Gaia DR1 positions for the brightest, unsaturated reference stars have positional uncertainties on the order of $\lesssim 1$ mas.

We selected reference background sources for the reregistration process by requiring that the sources be detected in all epochal coadds, have a $\mathrm{S} / \mathrm{N}>100$, and have positional uncertainties within $2 \sigma$ of the median positional uncertainty of the field. Requiring a detection in every coadded frame cut sources on the extreme edge of the field, while the positional uncertainty cut removed any sources with any significant proper motion. We then evaluated each reference target by eye to discard any non-pointlike sources. We obtained Gaia coordinates for each reference star, where available, and excluded any with exceptionally high uncertainties ( $\gtrsim 1$ mas) in the Gaia DR1, as well as reference sources that were lacking Gaia coordinates. The resulting set of reference stars varied from 7-96, depending on the stellar density in the field. Thumbnail images of three example fields with the target and reference sources highlighted can be found in Figure 3, showcasing fields with low, moderate, and high numbers of reference stars.

\subsection{Positional Uncertainties}

We found that the positional uncertainties output by the MOPEX/APEX centroid extractions were overestimated by a factor of $\gtrsim 2$ compared to the uncertainties on background stars with similar S/N. Instead of using these inflated uncertainties, we determined empirical positional uncertainties for each target by comparison to the positional uncertainties of the presumably non-moving field reference stars. For each field, we reregistered the locations of all stars in the field using the correction determined by the reference field stars as detailed above. We then calculated the positional uncertainty of every source in both R.A. and decl. as the standard deviation of the centroid location across all epochs, post-reregistration. As 
Table 5

Best-fit Astrometric Solutions

\begin{tabular}{|c|c|c|c|c|c|c|c|c|c|c|c|c|}
\hline $\begin{array}{l}\text { Object } \\
\text { Name } \\
(1)\end{array}$ & $\begin{array}{c}\alpha_{0,2014} \\
\text { (Deg, J2000) } \\
\text { (2) }\end{array}$ & $\begin{array}{c}\delta_{0,2014} \\
(\mathrm{Deg}, \mathrm{J} 2000) \\
(3)\end{array}$ & $\begin{array}{c}\sigma_{\alpha_{0}} \\
(\mathrm{mas}) \\
(4)\end{array}$ & $\begin{array}{c}\sigma_{\delta_{0}} \\
(\mathrm{mas}) \\
(5)\end{array}$ & $\begin{array}{c}\mu_{\alpha} \\
\left(\operatorname{mas~yr}^{-1}\right) \\
(6)\end{array}$ & $\begin{array}{c}\mu_{\delta} \\
\left(\operatorname{mas~yr}^{-1}\right) \\
(7)\end{array}$ & $\begin{array}{c}\pi_{\text {trig }} \\
\text { (mas, relative) } \\
(8)\end{array}$ & $\begin{array}{c}\text { Distance } \\
\text { (pc) } \\
(9)\end{array}$ & $\begin{array}{c}\sigma_{\mathrm{pos}} \\
(\mathrm{mas}) \\
(10)\end{array}$ & $\begin{array}{c}n_{\text {epochs }} \\
\text { (11) }\end{array}$ & $\begin{array}{l}n_{\text {ref }} \\
(12)\end{array}$ & $\begin{array}{c}\chi^{2} / \text { dof }=\chi_{\nu}^{2} \\
\text { (13) }\end{array}$ \\
\hline WISE $0146+4234$ & 26.735579 & 42.569408 & 8.69 & 6.24 & $-450.67 \pm 6.29$ & $-27.90 \pm 6.34$ & $45.575 \pm 5.74$ & $21.94_{-2.45}^{+3.16}$ & 20 & 15 & 18 & $34.0 / 25=1.36$ \\
\hline WISE $0336-0143$ & 54.020862 & -1.732170 & 6.65 & 6.48 & $-247.35 \pm 6.05$ & $-1213.46 \pm 6.03$ & $100.90 \pm 5.86$ & $9.91_{-0.54}^{+0.61}$ & 20 & 14 & 13 & $29.44 / 23=1.28$ \\
\hline WISE $0350-5658$ & 57.500996 & -56.975638 & 17.80 & 9.66 & $-206.94 \pm 6.52$ & $-577.67 \pm 6.68$ & $168.84 \pm 8.53$ & $5.92_{-0.28}^{+0.32}$ & 30 & 14 & 8 & $23.69 / 23=1.03$ \\
\hline WISE $0359-5401$ & 59.891827 & -54.032517 & 11.96 & 6.90 & $-152.70 \pm 4.83$ & $-783.66 \pm 4.93$ & $75.36 \pm 6.62$ & $13.27_{-1.07}^{+1.28}$ & 25 & 16 & 8 & $31.32 / 27=1.16$ \\
\hline WISE $0410+1502$ & 62.595853 & 15.044417 & 5.00 & 4.71 & $959.86 \pm 3.57$ & $-2218.64 \pm 3.46$ & $153.42 \pm 4.05$ & $6.52_{-0.17}^{+0.18}$ & 15 & 16 & 12 & $31.32 / 27=1.16$ \\
\hline WISE $0535-7500$ & 83.819477 & -75.006740 & 39.31 & 10.08 & $-113.23 \pm 7.71$ & $23.72 \pm 7.52$ & $79.51 \pm 8.79$ & $12.58_{-1.25}^{+1.56}$ & 30 & 12 & 28 & $32.3 / 19=1.70$ \\
\hline WISE 0647-6232 & 101.846784 & -62.542832 & 14.92 & 6.74 & $1.015 \pm 5.08$ & $390.97 \pm 4.61$ & $83.73 \pm 5.68$ & $11.94_{-0.76}^{+0.87}$ & 20 & 13 & 21 & $24.43 / 21=1.16$ \\
\hline WISE 0713-2917 & 108.344414 & -29.298188 & 5.51 & 4.72 & $341.10 \pm 6.57$ & $-411.13 \pm 6.00$ & $100.73 \pm 4.74$ & $9.93_{-0.45}^{+0.49}$ & 15 & 13 & 68 & $9.87 / 21=0.47$ \\
\hline WISE $0734-7157$ & 113.681539 & -71.962325 & 32.07 & 9.94 & $-566.22 \pm 8.85$ & $-77.54 \pm 8.82$ & $67.63 \pm 8.68$ & $14.79_{-1.68}^{+2.18}$ & 30 & 12 & 26 & $21.59 / 19=1.14$ \\
\hline WISE $0825+2805$ & 126.280554 & 28.096545 & 5.40 & 4.72 & $-64.35 \pm 5.56$ & $-234.73 \pm 5.36$ & $139.02 \pm 4.33$ & $7.19_{-0.22}^{+0.23}$ & 15 & 14 & 13 & $24.25 / 23=1.05$ \\
\hline WISE 1051-2138 & 162.875233 & -21.650040 & 6.74 & 6.28 & $145.57 \pm 6.84$ & $-160.68 \pm 6.60$ & $49.27 \pm 6.47$ & $20.3_{-2.4}^{+3.1}$ & 20 & 12 & 7 & $17.79 / 19=0.94$ \\
\hline WISE $1055-1652$ & 163.972546 & -16.870930 & 6.98 & 6.48 & $-1001.7 \pm 9.2$ & $432.16 \pm 9.17$ & $71.21 \pm 6.82$ & $14.04_{-1.2}^{+1.5}$ & 20 & 10 & 12 & $22.8 / 15=1.52$ \\
\hline WISE $1206+8401$ & 181.512553 & 84.019282 & 93.16 & 9.09 & $-557.69 \pm 6.54$ & $-241.31 \pm 6.51$ & $85.12 \pm 9.27$ & $11.75_{-1.15}^{+1.44}$ & 30 & 14 & 7 & $19.46 / 14=1.39$ \\
\hline WISE $1318-1758$ & 199.641070 & -17.974002 & 7.37 & 6.97 & $-514.59 \pm 7.20$ & $3.70 \pm 6.86$ & $48.06 \pm 7.33$ & $20.81_{-2.75}^{+3.74}$ & 25 & 15 & 7 & $30.79 / 15=1.23$ \\
\hline WISE $1405+5534$ & 211.322480 & 55.572793 & 14.60 & 8.54 & $-2336.04 \pm 6.91$ & $238.02 \pm 7.40$ & $144.35 \pm 8.60$ & $6.93_{-0.39}^{+0.44}$ & 25 & 13 & 7 & $32.84 / 21=1.56$ \\
\hline WISE $1541-2250$ & 235.464061 & -22.840554 & 5.03 & 4.51 & $-895.05 \pm 4.68$ & $-94.73 \pm 4.66$ & $167.05 \pm 4.19$ & $5.99_{-0.147}^{+0.154}$ & 15 & 15 & 26 & $24.38 / 25=0.98$ \\
\hline WISE 1639-6847 & 249.921736 & -68.797280 & 22.37 & 7.68 & $579.09 \pm 12.52$ & $-3104.54 \pm 12.25$ & $228.05 \pm 8.93$ & $4.39_{-0.17}^{+0.18}$ & 25 & 12 & 96 & $23.94 / 19=1.26$ \\
\hline WISE $1738+2732$ & 264.648443 & 27.549315 & 5.32 & 4.59 & $343.27 \pm 3.45$ & $-340.63 \pm 3.35$ & $136.26 \pm 4.27$ & $7.34_{-0.22}^{+0.24}$ & 15 & 16 & 13 & $28.95 / 27=1.07$ \\
\hline WISE $1828+2650$ & 277.130717 & 26.844012 & 5.04 & 4.58 & $1020.99 \pm 3.20$ & $175.55 \pm 3.09$ & $100.21 \pm 4.23$ & $9.98_{-0.40}^{+0.44}$ & 15 & 16 & 31 & $30.58 / 27=1.13$ \\
\hline WISE $2056+1459$ & 314.121287 & 14.9986666 & 4.54 & 4.39 & $822.99 \pm 3.37$ & $535.72 \pm 3.36$ & $138.32 \pm 3.86$ & $7.23_{-0.20}^{+0.21}$ & 15 & 16 & 38 & $18.19 / 27=0.67$ \\
\hline WISE $2209+2711$ & 332.275281 & 27.194171 & 6.58 & 5.95 & $1199.55 \pm 4.94$ & $-1359.00 \pm 4.76$ & $154.41 \pm 5.67$ & $6.48_{-0.23}^{+0.25}$ & 20 & 15 & 15 & $23.41 / 25=0.94$ \\
\hline WISE $2220-3628$ & 335.230875 & -36.471639 & 7.61 & 6.00 & $292.91 \pm 7.43$ & $-61.46 \pm 7.04$ & $84.10 \pm 5.90$ & $11.89_{-0.78}^{+0.90}$ & 20 & 14 & 13 & $22.71 / 23=0.99$ \\
\hline
\end{tabular}

(This table is available in machine-readable form.) 

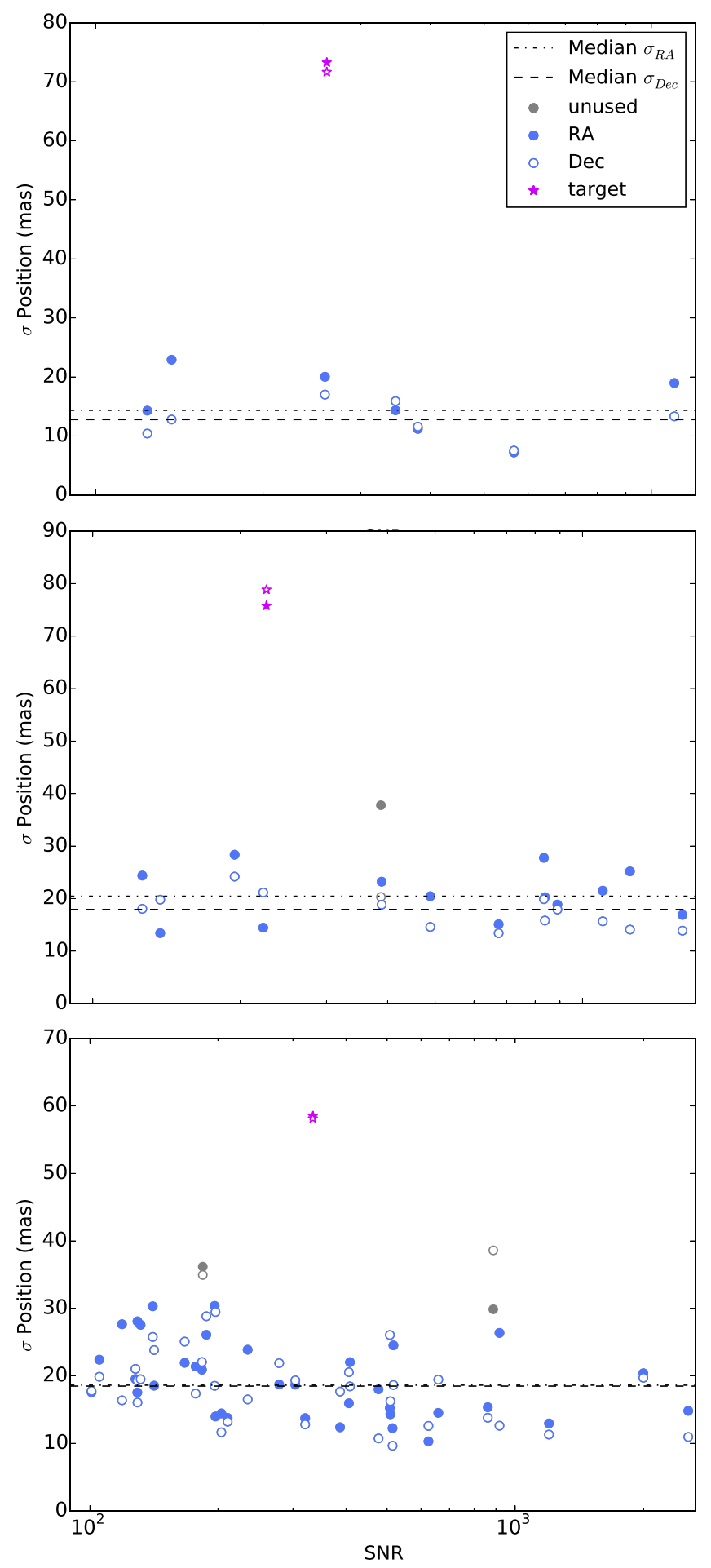

Figure 4. Positional uncertainties vs. $\mathrm{S} / \mathrm{N}$ for stars in the fields of WISE 1051 -2138 , WISE $2209+2711$, and WISE $1828+2650$, from left to right. Reference stars $\sigma_{\text {R.A. }}$ are in red and $\sigma_{\text {decl. }}$ are in blue. Uncertainties were calculated by taking the standard deviation of the centroid location across all epochs, post-reregistration. Positional uncertainty drops with increasing $\mathrm{S} / \mathrm{N}$, until reaching a systematic floor. We measure the median positional uncertainty for reference stars with a $\mathrm{S} / \mathrm{N}>100$ after performing a $1 \sigma$ clipping to remove outliers. The median value (horizontal lines) is used as the target positional uncertainty, in lieu of the MOPEX-given $\sigma_{\text {R.A. and }} \sigma_{\text {decl. }}$ (stars), which significantly overestimates the positional uncertainties.

expected, positional uncertainty drops with increasing $\mathrm{S} / \mathrm{N}$ until it reaches a systematic floor of $\sim 15-40$ mas, depending on the field. Figure 4 shows three examples of positional uncertainty versus source $\mathrm{S} / \mathrm{N}$, given a low, medium, or high number of reference stars. Our target $\mathrm{S} / \mathrm{Ns}$ are typically high enough that their positional uncertainties can be determined from the asymptotic portion of the graph. We measure the median positional uncertainty above a cutoff $\mathrm{S} / \mathrm{N}>100$ after performing a $2 \sigma$ clipping to remove significant outliers. These outliers could be non-pointlike sources, e.g., galaxies, or they could have significant proper motion. The median value rounded to the nearest 5 mas is then the positional uncertainty that we use in each epoch to determine the astrometric fits for each of our targets, with a floor of 15 mas. Positional uncertainties for each target are listed in Column 10 of Table 5.

After determining our target uncertainties, the selected reference stars that likewise met the sigma clipping requirement were then used to perform a final reregistration. We performed a least-squares affine transformation to adjust each frame onto the Gaia reference frame. To do this, we projected both the Gaia and MOPEX coordinates onto a tangent plane $(\xi, \eta)$ and then solved for the best-fit generalized six-term solution, allowing for offsets, rotation, and scaling between the two planes.

\subsection{Astrometric Solutions}

After reregistering each source onto a common reference frame, we then solved simultaneously for five parameters: trigonometric parallax $\pi$, proper motion in both R.A. and decl. $\left(\mu_{\alpha}, \mu_{\delta}\right)$, and initial position $\left(\alpha_{0}, \delta_{0}\right)$ at a fiducial time of $T_{0}=2014.0$, which falls roughly in the middle of our time baseline for each object. We used the standard astrometric equations (Smart \& Green 1977; Green 1985), inputting epochal coordinates, time of observation, and rectangular observatory coordinates obtained from the image headers. We then used Pythons' Scipy least-squares minimization module ${ }^{23}$ to solve for the best fit. Our best-fit astrometric solutions are listed in Table 5 and plotted in Figure 5. Three examples are printed here. The complete figure set ( 22 objects) is available in the online journal. We show both the overall astrometric fit, as well as the parallactic ellipse, after removing the best-fit proper motion component. The best-fit model shown makes use of the Spitzer ephemerides from JPL's Horizons ${ }^{24}$ to calculate the heliocentric rectangular coordinates of Spitzer over a longer time baseline and with higher cadence than our observations. These measurements are for relative parallaxes, not absolute. We estimate that the correction for the systematic offset of the average parallax of the background stars is $\sim 1$ mas, well within the random errors of our solutions.

One caveat for the targets at high declination $\left(|\delta| \gtrsim 70^{\circ}\right)$ is that an unidentifiable problem in the MOPEX mosaicking code leads to much more uncertain astrometry. This is reflected in the larger uncertainties we adopt for their epochal positions and the generally larger reduced chi-squared $\left(\chi_{\nu}^{2}\right)$ values we measure. This issue will be further discussed in our forthcoming paper presenting parallaxes for all of the T6 and later brown dwarfs in our parallax program (Kirkpatrick et al. 2018).

\footnotetext{
23 https://docs.scipy.org/doc/scipy-0.19.0/reference/generated/scipy.optimize. leastsq.html

${ }^{24}$ https://ssd.jpl.nasa.gov/?horizons
} 

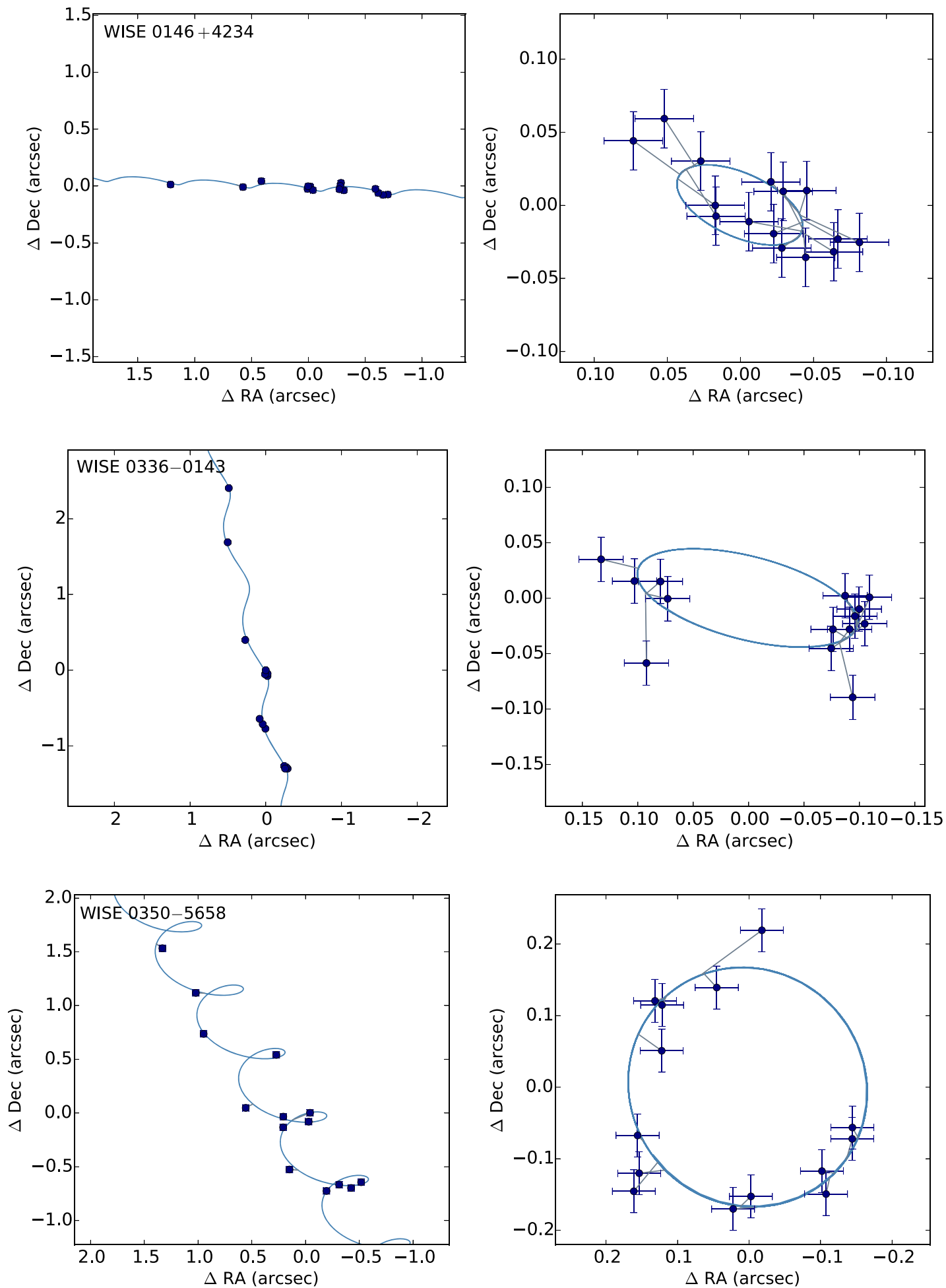

Figure 5. Astrometric fits for three of our targets. The complete figure set (22 objects) is available in the online journal. We maintained a square scaling for the $\Delta$ decl. and $\Delta$ R.A. Our observations are plotted in navy and the best-fit astrometric model is plotted in light blue. The left plots include proper motion and parallax and the right plots have proper motion removed. Note the differing scales between the left and right plots. WISE 0146+4234 is an unresolved binary, which produces systematic offsets of our astrometry and causes the parallactic ellipse to appear smaller than it is.

(The complete figure set (22 images) is available.)

\section{Results}

\subsection{Color-Magnitude Diagrams (CMDs)}

We used our measured distances to calculate the absolute magnitudes for all objects in $J_{\mathrm{MKO}}, H_{\mathrm{MKO}}$, [3.6], [4.5], $W 1$, and
$W 2$, when available, listed in Table 6. In Figure 6, we plot four different CMDs, showing $M_{J}$ versus $J-W 2, M_{H}$ versus $H-W 2, M_{W 2}$ versus $J-W 2$, and $M_{[4.5]}$ versus [3.6]-[4.5]. Data from this paper are plotted as filled circles and data from the literature are open symbols (Tinney et al. 2014: circles; 
Table 6

Absolute Magnitudes

\begin{tabular}{|c|c|c|c|c|c|c|c|c|}
\hline $\begin{array}{l}\text { Object } \\
\text { Name } \\
\text { (1) }\end{array}$ & $\begin{array}{l}\pi_{\text {trig }} \\
(\mathrm{mas}) \\
(2)\end{array}$ & $\begin{array}{l}\text { Distance } \\
\text { (pc) } \\
(3)\end{array}$ & $\begin{array}{c}M_{J} \\
(\mathrm{mag}) \\
(4)\end{array}$ & $\begin{array}{c}M_{H} \\
(\mathrm{mag}) \\
(5)\end{array}$ & $\begin{array}{c}M_{[3.6]} \\
(\mathrm{mag}) \\
(6)\end{array}$ & $\begin{array}{c}M_{[4.5]} \\
(\mathrm{mag}) \\
(7)\end{array}$ & $\begin{array}{c}M_{W 1} \\
(\mathrm{mag}) \\
(8)\end{array}$ & $\begin{array}{c}M_{W 2} \\
\text { (mag) } \\
\text { (9) }\end{array}$ \\
\hline WISE $0146+4234$ & $45.575 \pm 5.74$ & $21.94_{-2.45}^{+3.16}$ & $17.69 \pm 0.37$ & $17.00 \pm 0.36$ & $15.65 \pm 0.29$ & $13.36 \pm 0.27$ & $>17.43$ & $13.38 \pm 0.28$ \\
\hline WISE $0336-0143$ & $100.90 \pm 5.86$ & $9.91_{-0.54}^{+0.61}$ & $>21.12$ & $>20.22$ & $17.22 \pm 0.15$ & $14.65 \pm 0.13$ & $18.47 \pm 0.49$ & $14.58 \pm 0.14$ \\
\hline WISE $0350-5658$ & $168.84 \pm 8.53$ & $5.92_{-0.28}^{+0.32}$ & $23.32 \pm 0.13$ & $23.40 \pm 0.17$ & $18.97 \pm 0.17$ & $15.85 \pm 0.11$ & $>19.84$ & $15.88 \pm 0.12$ \\
\hline WISE $0359-5401$ & $75.36 \pm 6.62$ & $13.27_{-1.07}^{+1.28}$ & $20.95 \pm 0.20$ & $21.41 \pm 0.22$ & $16.95 \pm 0.22$ & $14.74 \pm 0.19$ & $>18.42$ & $14.77 \pm 0.20$ \\
\hline WISE $0410+1502$ & $153.42 \pm 4.05$ & $6.52_{-0.17}^{+0.18}$ & $20.25 \pm 0.06$ & $20.83 \pm 0.07$ & $17.51 \pm 0.07$ & $15.08 \pm 0.06$ & $>19.10$ & $15.04 \pm 0.07$ \\
\hline WISE $0535-7500$ & $79.51 \pm 8.79$ & $12.58_{-1.25}^{+1.56}$ & $21.63 \pm 0.25$ & $22.84 \pm 0.42$ & $17.15 \pm 0.26$ & $14.62 \pm 0.24$ & $17.44 \pm 0.28$ & $14.41 \pm 0.24$ \\
\hline WISE $0647-6232$ & $83.73 \pm 5.68$ & $11.94_{-0.76}^{+0.87}$ & $22.47 \pm 0.16$ & $22.92 \pm 0.22$ & $17.44 \pm 0.20$ & $14.77 \pm 0.15$ & $>19.15$ & $14.84 \pm 0.16$ \\
\hline WISE $0713-2917$ & $100.73 \pm 4.74$ & $9.93_{-0.45}^{+0.49}$ & $20.00 \pm 0.11$ & $20.21 \pm 0.13$ & $16.66 \pm 0.11$ & $14.22 \pm 0.10$ & $>18.79$ & $14.48 \pm 0.11$ \\
\hline WISE $0734-7157$ & $67.63 \pm 8.68$ & $14.79_{-1.68}^{+2.18}$ & $19.50 \pm 0.28$ & $20.22 \pm 0.29$ & $16.76 \pm 0.30$ & $14.42 \pm 0.28$ & $17.90 \pm 0.40$ & $14.34 \pm 0.28$ \\
\hline WISE $0825+2805$ & $139.02 \pm 4.33$ & $7.19_{-0.22}^{+0.23}$ & $23.12 \pm 0.08$ & $23.68 \pm 0.15$ & $18.14 \pm 0.12$ & $15.36 \pm 0.07$ & $>19.16$ & $15.29 \pm 0.09$ \\
\hline WISE $1051-2138$ & $49.27 \pm 6.47$ & $20.3_{-2.4}^{+3.1}$ & $17.40 \pm 0.30$ & $17.65 \pm 0.48$ & $14.93 \pm 0.29$ & $13.10 \pm 0.29$ & $15.76 \pm 6.84$ & $13.06 \pm 0.29$ \\
\hline WISE $1055-1652$ & $71.21 \pm 6.82$ & $14.04_{-1.2}^{+1.5}$ & $19.97 \pm 0.30$ & $>19.36$ & $16.61 \pm 0.22$ & $14.27 \pm 0.21$ & $>17.37$ & $14.33 \pm 0.22$ \\
\hline WISE $1206+8401$ & $85.12 \pm 9.27$ & $11.75_{-1.15}^{+1.44}$ & $20.12 \pm 0.24$ & $20.71 \pm 0.24$ & $16.91 \pm 0.25$ & $14.97 \pm 0.24$ & $>18.38$ & $14.71 \pm 0.24$ \\
\hline WISE $1318-1758$ & $48.06 \pm 7.33$ & $20.81_{-2.75}^{+3.74}$ & $16.84 \pm 0.38$ & $16.12 \pm 0.40$ & $15.20 \pm 0.34$ & $13.12 \pm 0.33$ & $15.92 \pm 0.37$ & $13.07 \pm 0.34$ \\
\hline WISE $1405+5534$ & $144.35 \pm 8.60$ & $6.93_{-0.39}^{+0.44}$ & $21.86 \pm 0.13$ & $22.30 \pm 0.15$ & $17.65 \pm 0.14$ & $14.87 \pm 0.13$ & $19.56 \pm 0.42$ & $14.89 \pm 0.13$ \\
\hline WISE $1541-2250$ & $167.05 \pm 4.19$ & $5.99_{-0.147}^{+0.154}$ & $22.75 \pm 0.08$ & $23.20 \pm 0.18$ & $17.63 \pm 0.07$ & $15.34 \pm 0.06$ & $17.85 \pm 0.17$ & $15.36 \pm 0.08$ \\
\hline WISE $1639-6847$ & $228.05 \pm 8.93$ & $4.39_{-0.17}^{+0.18}$ & $22.42 \pm 0.09$ & $22.54 \pm 0.09$ & $18.08 \pm 0.09$ & $15.47 \pm 0.09$ & $19.06 \pm 0.21$ & $15.33 \pm 0.10$ \\
\hline WISE $1738+2732$ & $136.26 \pm 4.27$ & $7.34_{-0.22}^{+0.24}$ & $20.22 \pm 0.07$ & $20.92 \pm 0.07$ & $17.64 \pm 0.09$ & $15.15 \pm 0.07$ & $18.38 \pm 0.17$ & $15.17 \pm 0.08$ \\
\hline WISE $1828+2650$ & $100.21 \pm 4.23$ & $9.98_{-0.40}^{+0.44}$ & $23.48 \pm 0.25$ & $22.85 \pm 0.26$ & $16.91 \pm 0.09$ & $14.33 \pm 0.09$ & $>18.25$ & $14.36 \pm 0.10$ \\
\hline WISE $2056+1459$ & $138.32 \pm 3.86$ & $7.23_{-0.20}^{+0.21}$ & $19.83 \pm 0.06$ & $20.35 \pm 0.07$ & $16.77 \pm 0.07$ & $14.61 \pm 0.06$ & $17.18 \pm 0.10$ & $14.54 \pm 0.07$ \\
\hline WISE $2209+2711$ & $154.41 \pm 5.67$ & $6.48_{-0.23}^{+0.25}$ & $23.80 \pm 0.15$ & $23.33 \pm 0.17$ & $18.68 \pm 0.14$ & $15.68 \pm 0.08$ & $>19.77$ & $15.71 \pm 0.10$ \\
\hline WISE $2220-3628$ & $84.10 \pm 5.90$ & $11.89_{-0.78}^{+0.90}$ & $20.07 \pm 0.15$ & $20.48 \pm 0.16$ & $16.80 \pm 0.17$ & $14.37 \pm 0.15$ & $>18.40$ & $14.34 \pm 0.16$ \\
\hline
\end{tabular}

(This table is available in machine-readable form.)

Dupuy \& Kraus 2013: diamonds). Every object is colored according to its spectral type, as shown in the legend. The CMDs show a tight trend, particularly in $M_{J}$ versus $J-W 2$ and $M_{H}$ versus $H-W 2$, in which the trends previously seen for earlier spectral types are continued, showing decreasing absolute magnitudes in the near-infrared as $J-W 2$ colors redden.

We determined a weighted linear fit to both $M_{J}$ versus $J-W 2$ and $M_{H}$ versus $H-W 2$ and tabulate the coefficients in Table 7. Although these relations require two photometric observations to obtain a photometric distance estimate, we find that this relationship is much tighter than if we were to determine fits to the absolute magnitude versus spectral type.

$M_{W 2}$ versus $J-W 2$ shows more scatter than the nearinfrared CMDs. Interestingly, $M_{W 2}$ versus $J-W 2$ appears to plateau in $M_{W 2}$ across the $\mathrm{T} / \mathrm{Y}$ transition. It is unclear if this feature is real, or due to a bias (systematic or otherwise). It is possible that this represents a $\mathrm{T} / \mathrm{Y}$ transition, perhaps due to the rainout of an opacity source or the appearance of the salt/ sulfide clouds (Morley et al. 2012).

The $M_{\text {[4.5] }}$ versus [3.6]-[4.5] plot shows significantly more cosmic scatter than the other panels in Figure 6. This is likely due to [3.6] being a nonideal band for observing objects with significant $\mathrm{CH}_{4}$ absorption. The blue tail of the $4.5 \mu \mathrm{m}$ bandpass falls into the [3.6] filter transmission, giving late- $\mathrm{T}$ and $\mathrm{Y}$ an overall very red slope in [3.6]. It is possible that variations in gravity and/or metallicity cause this slope to shift, producing the observed scatter. It is likely that the $W 2$ versus $W 1-W 2$ CMD would show a much tighter correlation, because the $W 1$ and $W 2$ bandpasses were designed specifically for cold brown dwarfs; however, many targets only have limits on their $W 1$ magnitudes.

\subsection{Absolute Magnitude versus SpT}

Figure 7 shows absolute magnitude in various near and midinfrared bands as a function of spectral type, for this sample as well as other values taken from the literature. Studying the relationship of the absolute magnitude emitted at each bandpass as a function of spectral type provides us with insight on the evolution of the brown dwarf spectral energy distribution as it cools over time. Earlier-type brown dwarfs tend to follow a narrow trend in absolute magnitude, with flux decreasing in each of the bands monotonically as a function of spectral type. Because spectral typing historically sorts objects by effective temperatures, we expected the Y dwarf sample to continue this trend. However, instead of a tight correlation between spectral type and absolute magnitude, we see a large amount of scatter, spanning as much as $\sim 5 \mathrm{mag}$ within the $\mathrm{Y} 0$ spectral class alone.

Such a large spread in absolute properties cannot be explained by typical levels of variability (Cushing et al. 2016; Leggett et al. 2016) and must be indicative of a different physical mechanism. In Figure 7, each of the objects is colored according to $J-W 2$ color cutoffs, as detailed in the legend. Here, we are using $J-W 2$ as a proxy for temperature, based on Figure 18 from Schneider et al. (2015), which in turn utilizes the atmospheric models of Saumon et al. (2012), and Morley et al. (2012, 2014). Regardless of the type of clouds used in the atmospheric models, they all show a monotonic reddening of $J-W 2$ as temperature decreases. When we 

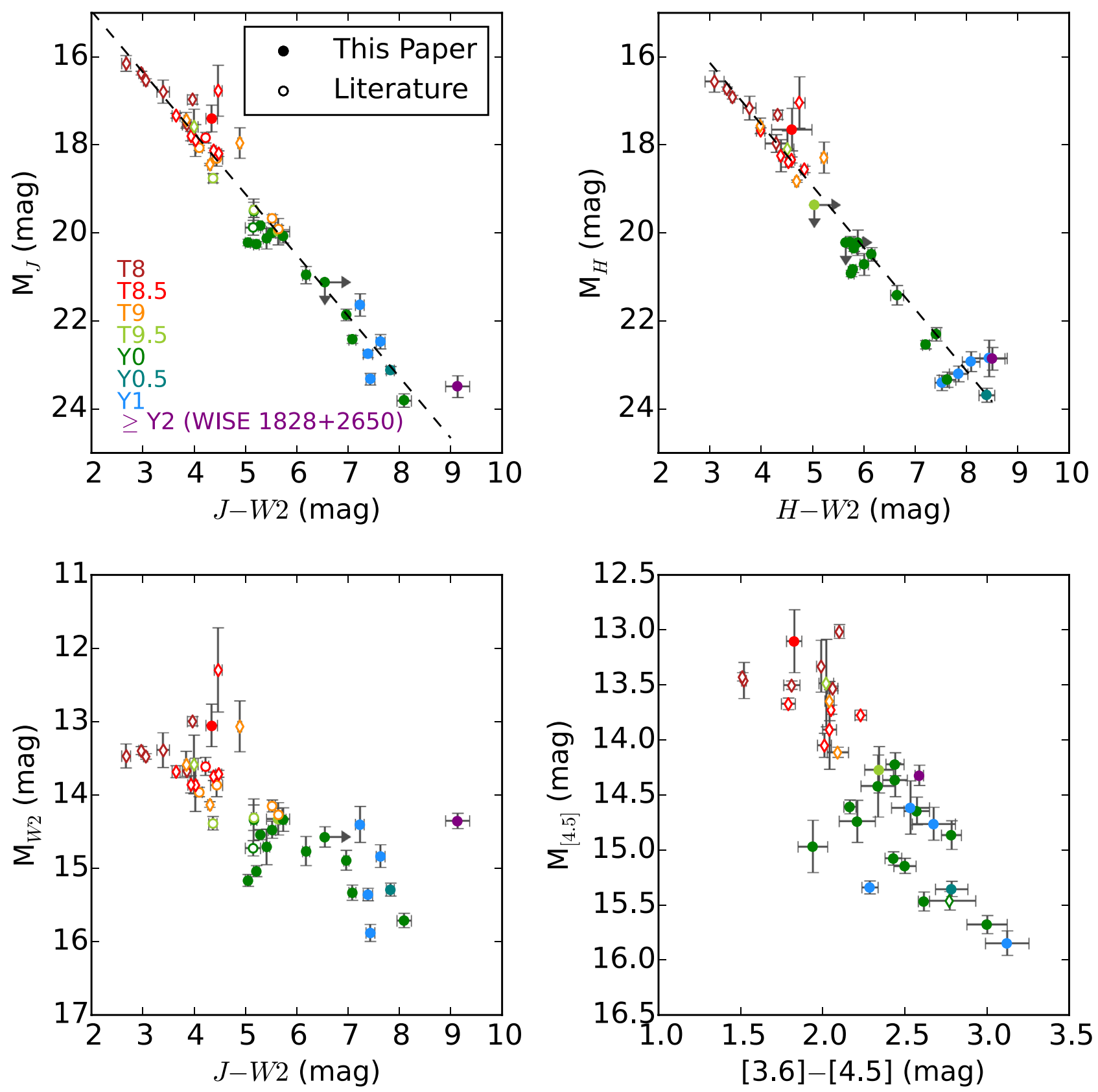

Figure 6. CMDs for $M_{J}$ vs. $J-W 2, M_{H}$ vs. $H-W 2, M_{W 2}$ vs. $J-W 2$, and $M_{[4.5]}$ vs. [3.6]-[4.5]. Open circles are from Tinney et al. (2014) and open diamonds are from Dupuy \& Kraus (2013). Filled circles are from this paper. Objects are shaded according to the spectral types listed in the legend. Weighted linear fits to $M_{J}$ vs. $J-W 2$ and $M_{H}$ vs. $H-W 2$ are plotted in dashed black lines.

Table 7

Coefficients for Linear Fits to Color-Magnitude Relations

\begin{tabular}{lccc}
\hline \hline Color & $\mathrm{c} 0$ & $\mathrm{c} 1$ & $\mathrm{rms}$ \\
$(1)$ & $(2)$ & $(3)$ & $(4)$ \\
\hline$M_{J}$ versus $J-W 2$ & 12.186 & 1.386 & 0.475 \\
$M_{H}$ versus $H-W 2$ & 11.935 & 1.401 & 0.544 \\
\hline
\end{tabular}

Note. These coefficients fit a line such that $M_{X}=c 0+c 1 \times\left(M_{X}-W 2\right)$, where $X$ is the $J$ or $H$ photometry on the MKO system.

separate objects by their $J-W 2$ color, new trends appear in Figure 7. In particular, the Y0 dwarfs appear to cover a very broad range in effective temperatures, likely accounting for the $\sim 5$ orders of absolute magnitudes observed in the $J$ band.

\subsection{Spectrophotometric and Photometric Distances for New Discoveries}

In Section 6.1 we determine photometric distance relationships based on linear fits to $M_{J}$ versus $J-W 2$ and $M_{H}$ versus $H-W 2$. These fits are valid for objects with $2<J-W 2<9$ and $3<H-W 2<9$. Below, we use this photometric distance relationship to estimate distances to the new $\geqslant \mathrm{T} 8$ objects presented here. For objects $<\mathrm{T} 8$, we use the spectrophotometric distance relations from Filippazzo et al. (2015).

WISE 0550-1950: We do not have an adequate baseline to measure the parallax of this new T6.5 dwarf, but using the spectrophotometric distance estimates from Filippazzo et al. (2015), we estimate a distance of $32.9 \mathrm{pc}$ to this object. 

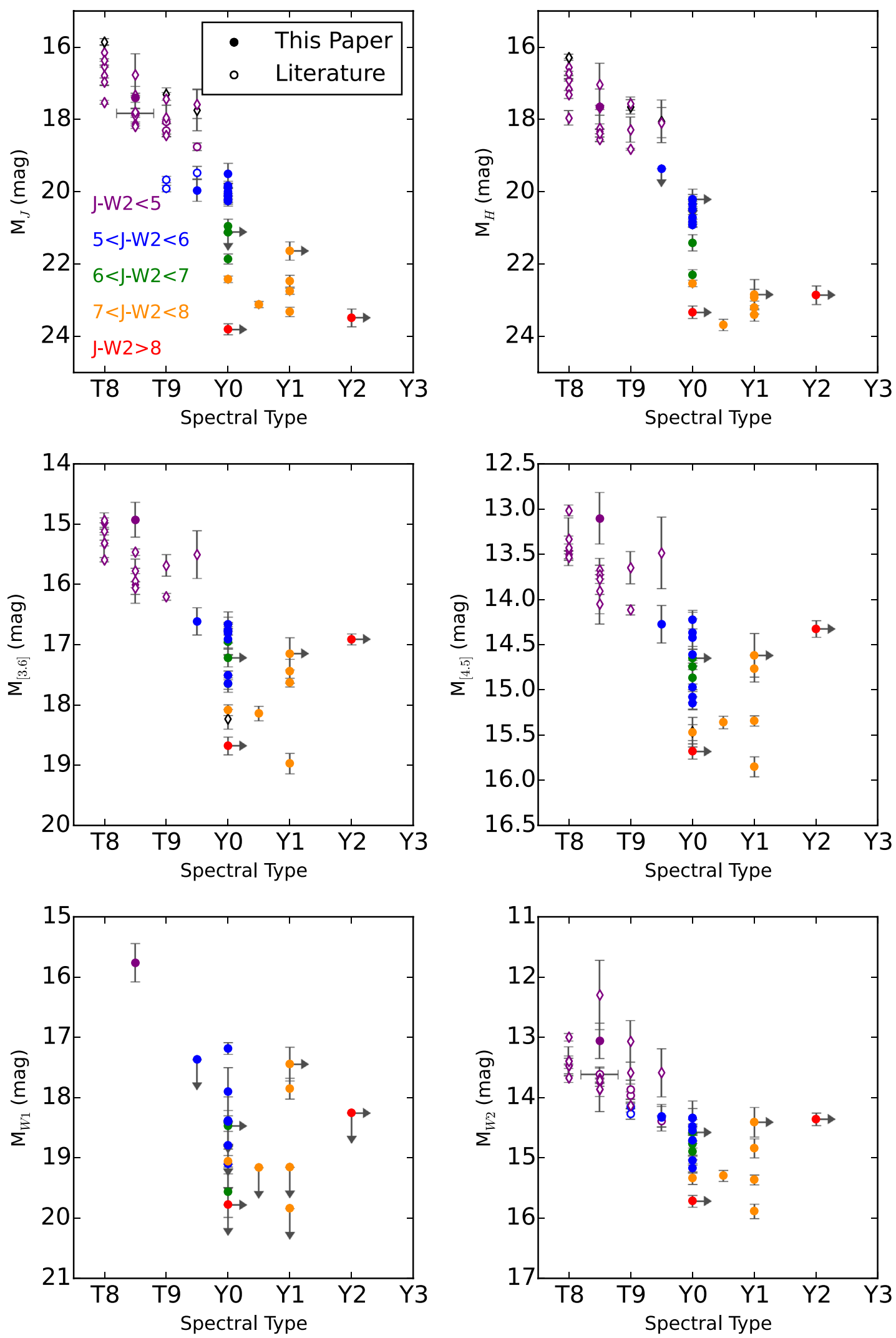

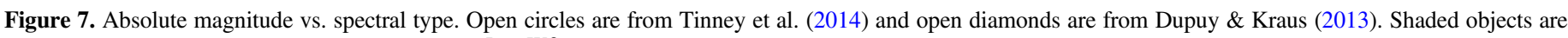
from this paper. Objects are shaded according to $J-W 2$ color, as shown in the legend.

WISE $0615+1526$ : We estimate a photometric distance of $22.3 \mathrm{pc}$ for this object.

WISE $0642+0423$ : We estimate its photometric distance to be $29.6 \mathrm{pc}$.
WISE 1220+5407: Our photometric distance estimate puts it at $22.5 \mathrm{pc}$.

WISE $2203+4619$ is estimated to be 18.9 pc away, based on our photometric distance relationships in Section 6.1. 
Table 8

Comparison to Published Parallaxes and Proper Motions

\begin{tabular}{|c|c|c|c|c|c|c|c|}
\hline $\begin{array}{l}\text { Object } \\
\text { (1) }\end{array}$ & $\begin{array}{c}\text { Measurement } \\
\text { (2) }\end{array}$ & $\begin{array}{l}\text { This Paper } \\
\text { (3) }\end{array}$ & $\begin{array}{c}\text { Smart et al. (2017) } \\
\text { (4) }\end{array}$ & $\begin{array}{c}\text { Beichman et al. (2014) } \\
\text { (5) }\end{array}$ & $\begin{array}{c}\text { Tinney et al. (2014) } \\
\text { (6) }\end{array}$ & $\begin{array}{c}\text { Dupuy \& Kraus (2013) } \\
\text { (7) }\end{array}$ & $\begin{array}{c}\text { Leggett et al. (2017) } \\
\text { (8) }\end{array}$ \\
\hline \multirow{3}{*}{ WISE $0146+4234$} & $\pi_{\text {trig }}$ (mas) & $45.6 \pm 5.7$ & 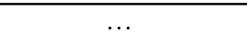 & $94 \pm 14$ & $\ldots$ & $\ldots$ & $54 \pm 5$ \\
\hline & $\mu_{\alpha}\left(\operatorname{mas~yr}^{-1}\right)$ & $-450.67 \pm 6.3$ & $\ldots$ & $-441 \pm 13$ & $\cdots$ & $\cdots$ & $-455 \pm 4$ \\
\hline & 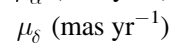 & $-27.9 \pm 6.3$ & $\cdots$ & $-26 \pm 16$ & $\cdots$ & $\ldots$ & $-24 \pm 4$ \\
\hline \multirow{3}{*}{ WISE $0350-5658$} & $\pi_{\text {trig }}(\mathrm{mas})$ & $168.8 \pm 8.5$ & $\cdots$ & $\ldots$ & $\cdots$ & $\cdots$ & $184 \pm 10$ \\
\hline & $\mu_{\alpha}\left(\operatorname{mas~yr}^{-1}\right)$ & $-206.9 \pm 6.5$ & $\cdots$ & $\cdots$ & $\cdots$ & $\cdots$ & $-206 \pm 7$ \\
\hline & $\mu_{\delta}\left(\operatorname{mas~yr}^{-1}\right)$ & $-577.7 \pm 6.7$ & $\cdots$ & $\cdots$ & $\cdots$ & $\cdots$ & $-578 \pm 8$ \\
\hline \multirow{3}{*}{ WISE $0359-5401$} & $\pi_{\text {trig }}(\mathrm{mas})$ & $75.4 \pm 6.62$ & $\cdots$ & $\cdots$ & $63.2 \pm 6.0$ & $\cdots$ & $\ldots$ \\
\hline & $\mu_{\alpha}\left(\operatorname{mas~yr}^{-1}\right)$ & $-152.7 \pm 4.8$ & $\cdots$ & $\ldots$ & $-176.0 \pm 10.8$ & $\ldots$ & $\ldots$ \\
\hline & $\mu_{\delta}\left(\operatorname{mas~yr}^{-1}\right)$ & $-783.7 \pm 4.9$ & $\cdots$ & $\cdots$ & $-744.5 \pm 11.9$ & $\cdots$ & $\cdots$ \\
\hline \multirow{3}{*}{ WISE $0410+1502$} & $\pi_{\text {trig }}(\mathrm{mas})$ & $153.4 \pm 4.0$ & $144.3 \pm 9.9$ & $160 \pm 9$ & $\ldots$ & $132 \pm 15$ & $\ldots$ \\
\hline & $\mu_{\alpha}\left(\operatorname{mas~yr}^{-1}\right)$ & $959.9 \pm 3.6$ & $956.8 \pm 5.6$ & $966 \pm 13$ & $\cdots$ & $958 \pm 37$ & $\ldots$ \\
\hline & $\mu_{\delta}\left(\operatorname{mas~yr}^{-1}\right)$ & $-2218.6 \pm 3.5$ & $-2221.2 \pm 5.5$ & $-2218 \pm 13$ & $\cdots$ & $-2229 \pm 29$ & $\cdots$ \\
\hline \multirow{3}{*}{ WISE $0535-7500$} & $\pi_{\text {trig }}(\mathrm{mas})$ & $79.5 \pm 8.8$ & $\ldots$ & $\ldots$ & $74 \pm 14$ & $\ldots$ & $70 \pm 5$ \\
\hline & $\mu_{\alpha}\left(\operatorname{mas~yr}^{-1}\right)$ & $-113.2 \pm 7.7$ & $\cdots$ & $\ldots$ & $-113.4 \pm 15.4$ & $\ldots$ & $-127 \pm 4$ \\
\hline & $\mu_{\delta}\left(\operatorname{mas~yr}^{-1}\right)$ & $23.7 \pm 7.5$ & $\ldots$ & $\ldots$ & $36.2 \pm 8.8$ & $\ldots$ & $13 \pm 4$ \\
\hline \multirow{3}{*}{ WISE 0647-6232 } & $\pi_{\text {trig }}(\mathrm{mas})$ & $83.7 \pm 5.7$ & $\ldots$ & $\ldots$ & $93 \pm 13$ & $\ldots$ & $\ldots$ \\
\hline & $\mu_{\alpha}\left(\operatorname{mas~yr}^{-1}\right)$ & $1.0 \pm 5.1$ & $\cdots$ & $\ldots$ & $0.6 \pm 16.1$ & $\cdots$ & $\ldots$ \\
\hline & $\mu_{\delta}\left(\operatorname{mas~yr}^{-1}\right)$ & $391.0 \pm 4.6$ & $\ldots$ & $\ldots$ & $368.0 \pm 18.0$ & $\ldots$ & $\ldots$ \\
\hline \multirow{3}{*}{ WISE 0713-2917 } & $\pi_{\text {trig }}$ (mas) & $100.7 \pm 4.7$ & $\ldots$ & $106 \pm 13$ & $08.7 \pm 4.0$ & $\ldots$ & $\ldots$ \\
\hline & $\mu_{\alpha}\left(\operatorname{mas~yr}^{-1}\right)$ & $341.1 \pm 6.6$ & $\ldots$ & $388 \pm 20$ & $350.1 \pm 4.8$ & $\ldots$ & $\ldots$ \\
\hline & 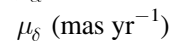 & $-411.1 \pm 6.0$ & $\ldots$ & $-419 \pm 22$ & $-411.4 \pm 5.6$ & $\cdots$ & $\ldots$ \\
\hline \multirow{3}{*}{ WISE $0734-7157$} & $\pi_{\text {trig }}$ (mas) & $67.6 \pm 8.7$ & $\ldots$ & $\ldots$ & $73.7 \pm 6.6$ & $\ldots$ & $\ldots$ \\
\hline & $\mu_{\alpha}\left(\operatorname{mas~yr}^{-1}\right)$ & $-566.2 \pm 8.8$ & $\ldots$ & $\ldots$ & $-565.8 \pm 7.7$ & $\ldots$ & $\ldots$ \\
\hline & $\mu_{\delta}\left(\operatorname{mas~yr}^{-1}\right)$ & $-77.5 \pm 8.8$ & $\cdots$ & $\cdots$ & $-81.5 \pm 8.0$ & $\cdots$ & $\ldots$ \\
\hline \multirow{3}{*}{ WISE $0825+2805$} & $\pi_{\text {trig }}(\mathrm{mas})$ & $139.0 \pm 4.3$ & $\ldots$ & $\cdots$ & $\cdots$ & $\cdots$ & $158 \pm 7$ \\
\hline & $\mu_{\alpha}\left(\operatorname{mas~yr}^{-1}\right)$ & $-64.4 \pm 5.6$ & $\ldots$ & $\ldots$ & $\cdots$ & $\ldots$ & $-66 \pm 8$ \\
\hline & $\mu_{\delta}\left(\operatorname{mas~yr}^{-1}\right)$ & $-234.7 \pm 5.4$ & $\ldots$ & $\cdots$ & $\cdots$ & $\cdots$ & $-247 \pm 10$ \\
\hline \multirow{3}{*}{ WISE $1206+8401$} & $\pi_{\text {trig }}(\mathrm{mas})$ & $85.1 \pm 9.3$ & $\cdots$ & $\cdots$ & $\cdots$ & $\cdots$ & $85 \pm 7$ \\
\hline & $\mu_{\alpha}\left(\operatorname{mas~yr}^{-1}\right)$ & $-557.7 \pm 6.5$ & $\ldots$ & $\ldots$ & $\ldots$ & $\ldots$ & $-585 \pm 4$ \\
\hline & $\mu_{\delta}\left(\operatorname{mas~yr}^{-1}\right)$ & $-241.3 \pm 6.5$ & $\cdots$ & $\cdots$ & $\cdots$ & $\cdots$ & $-253 \pm 5$ \\
\hline \multirow{3}{*}{ WISE $1405+5534$} & $\pi_{\text {trig }}(\mathrm{mas})$ & $144.3 \pm 8.6$ & $\ldots$ & $\ldots$ & $\cdots$ & $129 \pm 19$ & $155 \pm 6$ \\
\hline & $\mu_{\alpha}\left(\operatorname{mas~yr}^{-1}\right)$ & $-2336.0 \pm 6.9$ & $\ldots$ & $\ldots$ & $\ldots$ & $-2263 \pm 47$ & $-2334 \pm 5$ \\
\hline & $\mu_{\delta}\left(\operatorname{mas~yr}^{-1}\right)$ & $238.0 \pm 7.40$ & $\cdots$ & $\cdots$ & $\cdots$ & $288 \pm 41$ & $232 \pm 5$ \\
\hline \multirow[b]{2}{*}{ WISE $1541-2250$} & $\pi_{\text {trig }}(\mathrm{mas})$ & $167.1 \pm 4.2$ & $\ldots$ & $176 \pm 9$ & $175.1 \pm 4.4$ & $74 \pm 31$ & $\ldots$ \\
\hline & $\mu_{\alpha}\left(\operatorname{mas~yr}^{-1}\right)$ & $-895.0 \pm 4.7$ & $\ldots$ & $-857 \pm 12$ & $-894.7 \pm 4.2$ & $-870 \pm 130$ & $\ldots$ \\
\hline
\end{tabular}


Table 8

(Continued)

\begin{tabular}{|c|c|c|c|c|c|c|c|}
\hline $\begin{array}{l}\text { Object } \\
(1)\end{array}$ & $\begin{array}{l}\text { Measurement } \\
\text { (2) }\end{array}$ & $\begin{array}{l}\text { This Paper } \\
\text { (3) }\end{array}$ & $\begin{array}{l}\text { Smart et al. (2017) } \\
\text { (4) }\end{array}$ & $\begin{array}{l}\text { Beichman et al. (2014) } \\
\text { (5) }\end{array}$ & $\begin{array}{c}\text { Tinney et al. (2014) } \\
\text { (6) }\end{array}$ & $\begin{array}{l}\text { Dupuy \& Kraus (2013) } \\
\text { (7) }\end{array}$ & $\begin{array}{c}\text { Leggett et al. (2017) }{ }^{\mathrm{a}} \\
\text { (8) }\end{array}$ \\
\hline & $\mu_{\delta}\left(\operatorname{mas~yr}^{-1}\right)$ & $-94.7 \pm 4.7$ & 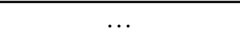 & $-87 \pm 13$ & $-87.7 \pm 4.7$ & $-13 \pm 58$ & 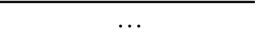 \\
\hline \multirow{3}{*}{ WISE $1639-6847$} & $\pi_{\text {trig }}(\mathrm{mas})$ & $228.1 \pm 8.9$ & $\cdots$ & $\cdots$ & $202.3 \pm 3.1$ & $\cdots$ & $\cdots$ \\
\hline & $\mu_{\alpha}\left(\operatorname{mas~yr}^{-1}\right)$ & $579.1 \pm 12.5$ & $\cdots$ & $\cdots$ & $586.0 \pm 5.5$ & $\cdots$ & $\cdots$ \\
\hline & $\mu_{\delta}\left(\operatorname{mas~yr}^{-1}\right)$ & $-3104.5 \pm 12.2$ & $\cdots$ & $\cdots$ & $-3101.1 \pm 3.6$ & $\cdots$ & $\cdots$ \\
\hline \multirow{3}{*}{ WISE $1738+2732$} & $\pi_{\text {trig }}(\mathrm{mas})$ & $136.3 \pm 4.3$ & $128.5 \pm 6.3$ & $128 \pm 10$ & $\cdots$ & $102 \pm 18$ & $\cdots$ \\
\hline & $\mu_{\alpha}\left(\operatorname{mas~yr}^{-1}\right)$ & $343.3 \pm 3.5$ & $345.0 \pm 5.7$ & $317 \pm 9$ & $\cdots$ & $292 \pm 63$ & $\cdots$ \\
\hline & $\mu_{\delta}\left(\operatorname{mas~yr}^{-1}\right)$ & $-340.6 \pm 3.4$ & $-340.1 \pm 5.1$ & $-321 \pm 11$ & $\cdots$ & $-396 \pm 22$ & $\cdots$ \\
\hline \multirow{3}{*}{ WISE $1828+2650$} & $\pi_{\text {trig }}($ mas) & $100.2 \pm 4.2$ & $\cdots$ & $106 \pm 7$ & $\cdots$ & $70 \pm 14$ & $\cdots$ \\
\hline & $\mu_{\alpha}\left(\operatorname{mas~yr}^{-1}\right)$ & $1021.0 \pm 3.2$ & $\cdots$ & $1024 \pm 7$ & $\cdots$ & $1020 \pm 15$ & $\cdots$ \\
\hline & $\mu_{\delta}\left({\left.\operatorname{mas~} \mathrm{yr}^{-1}\right)}^{-1}\right.$ & $175.6 \pm 3.1$ & $\cdots$ & $\ldots$ & $\cdots$ & $173 \pm 16$ & $\cdots$ \\
\hline \multirow{3}{*}{ WISE $2056+1459$} & $\pi_{\text {trig }}(\mathrm{mas})$ & $138.3 \pm 3.9$ & $148.9 \pm 8.2$ & $140 \pm 9$ & $\cdots$ & $144 \pm 23$ & $\cdots$ \\
\hline & $\mu_{\alpha}\left(\operatorname{mas~yr}^{-1}\right)$ & $823.0 \pm 3.3$ & $826.4 \pm 5.5$ & $812 \pm 9$ & $\cdots$ & $761 \pm 46$ & $\cdots$ \\
\hline & $\mu_{\delta}\left(\operatorname{mas} \mathrm{yr}^{-1}\right)$ & $535.7 \pm 3.4$ & $530.7 \pm 8.5$ & $34 \pm 8$ & $\ldots$ & $500 \pm 21$ & $\ldots$ \\
\hline \multirow{3}{*}{ WISE $2209+2711$} & $\pi_{\text {trig }}($ mas) & $154.4 \pm 5.7$ & $\cdots$ & $147 \pm 11$ & $\cdots$ & $\cdots$ & $\cdots$ \\
\hline & $\mu_{\alpha}\left(\operatorname{mas~yr}^{-1}\right)$ & $1199.6 \pm 4.9$ & $\cdots$ & $\mathrm{c} 1217 \pm 13$ & $\cdots$ & $\cdots$ & $\cdots$ \\
\hline & $\mu_{\delta}\left(\operatorname{mas~yr}^{-1}\right)$ & $-1359.0 \pm 4.8$ & $\cdots$ & $-1372 \pm 15$ & $\cdots$ & $\cdots$ & $\cdots$ \\
\hline \multirow{3}{*}{ WISE $2220-3628$} & $\pi_{\text {trig }}(\mathrm{mas})$ & $84.1 \pm 5.9$ & $\cdots$ & $136 \pm 17$ & $87.2 \pm 3.7$ & $\cdots$ & $\cdots$ \\
\hline & $\mu_{\alpha}\left(\operatorname{mas~yr}^{-1}\right)$ & $292.9 \pm 7.4$ & $\cdots$ & $283 \pm 13$ & $282.7 \pm 5.0$ & $\cdots$ & $\cdots$ \\
\hline & $\mu_{\delta}\left({\left.\operatorname{mas~} \mathrm{yr}^{-1}\right)}^{-1}\right.$ & $-61.5 \pm 7.0$ & $\cdots$ & $-97 \pm 17$ & $-94.0 \pm 3.0$ & $\cdots$ & $\cdots$ \\
\hline
\end{tabular}

Notes.

The data presented in Leggett et al. (2017) include astrometric data first published in Luhman \& Esplin (2016). 


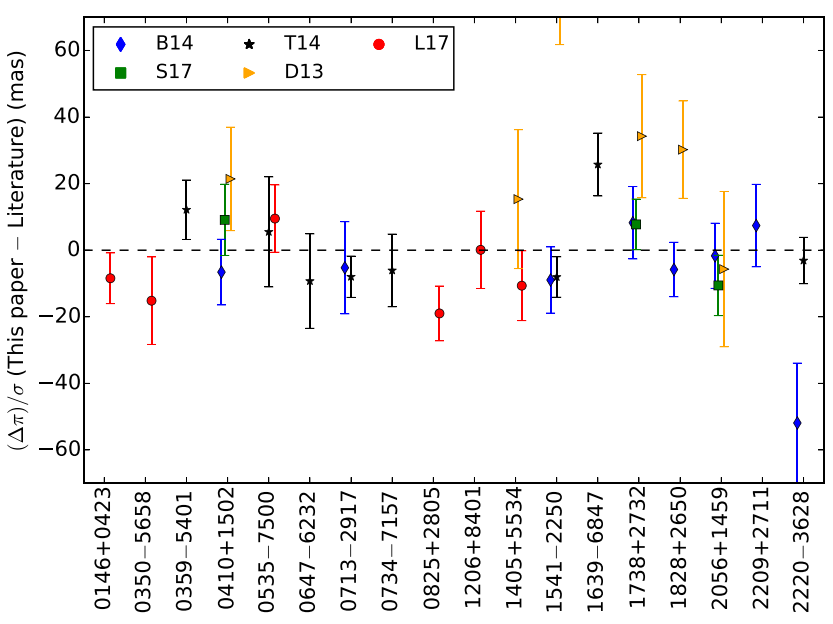

(a)

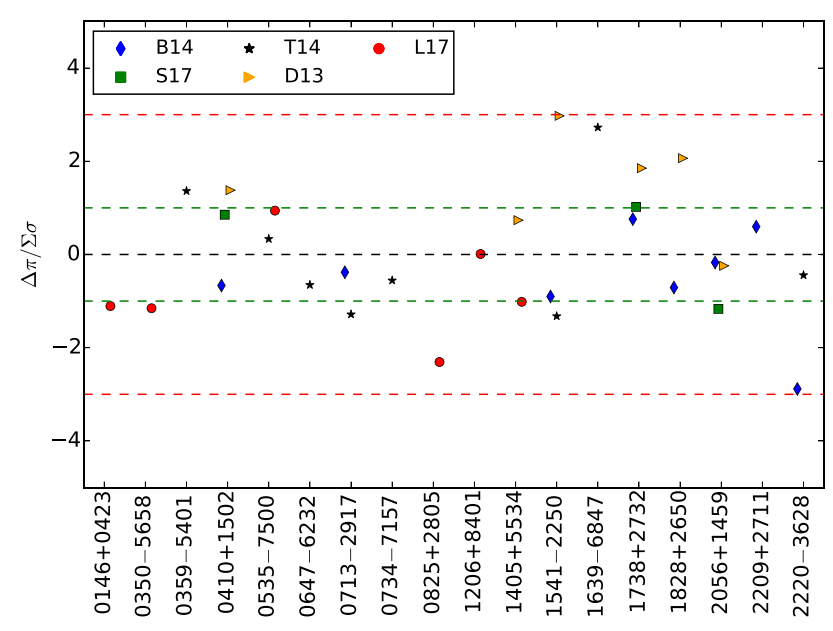

(b)

Figure 8. (a) Comparison of the difference in parallax values from this paper with the literature, vs. target name. Differences from Beichman et al. (2014) are in blue diamonds, Tinney et al. (2014) in black stars, Leggett et al. (2017) in red circles, Smart et al. (2017) in green squares, and Dupuy \& Kraus (2013) in yellow triangles. Note that the Dupuy \& Kraus (2013) value for 1541 is off the chart, their parallax being miscalculated due to a blend with a background star in their data set. (b) Fractional $\sigma$ difference between this paper and the literature. Dashed green lines denote $1 \sigma$ offsets and dashed red lines denote $3 \sigma$ offsets. With a few exceptions, our measured parallaxes are consistent within $1 \sigma$ to previously published values.

\subsection{Comparison to Literature}

In Table 8 and Figure 8 we compare our results to previously published astrometric fits for all of our targets with previous parallax measurements. We find that our results are mostly consistent with previously published values in the literature, with a few notable exceptions.

\subsubsection{Comparison to Tinney et al. (2014) and Beichman et al. (2014)}

Our results are consistent with Tinney et al. (2014) and Beichman et al. (2014) for most objects, with the exception of WISE 2220-3628. For this object, we find a consistent astrometric fit to the Tinney et al. (2014) data set, but our results are discrepant from those of Beichman et al. (2014). Upon further review of the Beichman et al. (2014) data set, we noticed that their measurements only cover one side of the parallactic ellipse, leaving the other side unconstrained and biasing the measurement. This is likely the cause of their discrepant fit.

\subsubsection{Comparison to Dupuy \& Kraus (2013)}

We also measure significant offsets in parallax values from Dupuy \& Kraus (2013). We find systematically larger parallax values (closer distances) than they do for 5 out of the 6 targets we have in common. Each object has at least one other measurement in the literature and we find that we are consistently in agreement with the other reference. Tinney et al. (2014) and Smart et al. (2017) also note systematic offsets between their parallax measurements and those of Dupuy \& Kraus (2013), concluding that these are likely due to the smaller number of measurements and thus a degeneracy between the parallax and proper motion parameters. For the extreme case of 1541-2250 (not plotted in comparison figures), we note, as did Beichman et al. (2014) and Tinney et al. (2014) that this object has several epochs skewed by a blend with a background star that throw off the fit in the [3.6] data, which explains the $>3-\sigma$ difference between the Dupuy \& Kraus (2013) results and others in the literature.

We explored several hypotheses to explain the discrepancies between the Dupuy \& Kraus (2013) measurements and those presented here. Similar to our parallax measurements, Dupuy \& Kraus (2013) uses the IRAC instrument on Spitzer to measure the positions of each target. However, they observed in [3.6], whereas the measurements presented here were made using [4.5] data.

Our first hypothesis is that the use of [3.6] data causes a chromatic distortion on the image plane that is different for the target than the background stars, and which would cause a systematic offset in the positions of the targets in the Dupuy \& Kraus (2013) data set. The IRAC instrument design utilizes beam splitters in each of its two fields of view to refract shorter wavelength light ([3.6] and [4.5]) to separate focal planes from the longer wavelength light (ch3 and ch4). Both [3.6] and [4.5] have similar background characteristics during the warm mission (Carey et al. 2010). The brown dwarf targets are significantly fainter in [3.6] compared to [4.5], requiring longer integration times (thus providing more background stars in each field). Late-T and $\mathrm{Y}$ dwarfs exhibit extreme methane absorption near the methane fundamental bandhead at $3.3 \mu \mathrm{m}$, which produces a dramatic upward slope in the spectral energy distribution within the [3.6] bandpass. Thus the targets have significantly redder effective central wavelengths compared to the relatively flat spectral energy distributions of the background stars. This reddening effect in [3.6] would lead to a slightly different average angle of refraction, compared to the background stars' average angles of refraction. The target spectral energy distributions in the [4.5] bandpass peak much closer to the center of the bandpass and should not have significantly different effective wavelengths from the background stars, so they should be immune to this effect. We thus expect that this effect would be evident by comparing the offset parallax measurement to the [3.6]-[4.5] color (Figure 9). We see a slight correlation between [3.6]-[4.5] color and parallax offset, but there is not enough data to draw a firm conclusion.

Our second hypothesis is that there is a fundamental difference between our fitting analysis and that of Dupuy \& Kraus (2013). In Section 2.4 of Dupuy \& Liu (2012), they describe their methodology for determining astrometric fits: 


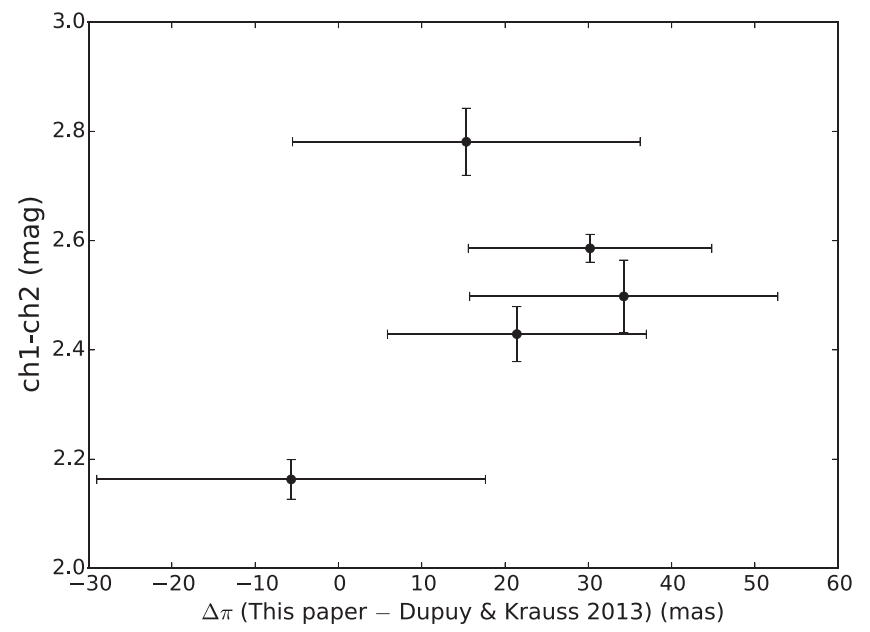

Figure 9. Comparison of parallax offset between our values and those of Dupuy \& Kraus (2013) vs. [3.6]-[4.5] color. If the extremely red-sloped [3.6] bandpass were responsible for the offset, we would expect to see an increasing trend in offset vs. [3.6]-[4.5] color. A slight correlation is seen, though there is not enough data to draw a firm conclusion.

"We fitted three parameters to the combined $(\alpha, \delta)$ data: proper motion in right ascension $\left(\mu_{\alpha}\right)$, proper motion in declination $\left(\mu_{\delta}\right)$, and parallax $(\pi)$. This is notably different from one standard approach taken in the literature of fitting two separate values of the parallax in $\alpha$ and $\delta(\ldots)$ MPFIT minimized the residuals in $(\alpha, \delta)$ after subtracting the relative parallax and proper motion offsets (three parameters) and the mean $(\alpha, \delta)$ position (effectively removing 2 additional degrees of freedom)."

We interpret this to meant that the subtraction of the average $(\alpha, \delta)$ position requires the parallax solution to fit through one point located at the center of the parallactic ellipse. The effect would be averaged out over long time baselines, but we believe this method to be ineffectual for limited epochs. The sense of the bias that we see is in the expected direction; that is, their ellipse fits are artificially smaller because of their choice of data analysis method. To test this, we performed a reduction of the same [3.6] data used in their paper but employing our methodology described above. In this case, we used the [3.6] PRF appropriate for Warm Spitzer data. The resulting astrometric fits are compared to our [4.5] parallax measurements in Figure 10. In Figure 10, the original measurements from Dupuy \& Kraus (2013) are shown in yellow, and the recalculation using our fitting analysis and the Dupuy \& Kraus (2013) data are in blue. In most cases, our calculations measure parallax solutions that are closer to those measured with our [4.5] data, though consistent with the original Dupuy \& Kraus (2013) values within the uncertainties.

Our third hypothesis to explain the discrepant measurements is that the shorter time baseline of the Dupuy \& Kraus (2013) data set made it difficult to disentangle the effects of proper motion when calculating the parallax. We explored this effect by reducing later epochs of [3.6] data, available on the Spitzer archive. The addition of 9-10 epochs for each target cannot fully account for the earlier difference seen between the [3.6] and [4.5] parallax measurements. These differences are plotted in Figure 10 in red. All targets except for $0410+1502$ show an improved comparison, though the systematic offset remains.

We believe that some combination of the three effects contributed to the systematically offset parallaxes published in Dupuy \& Kraus (2013). After re-reducing the Dupuy \& Kraus (2013) data

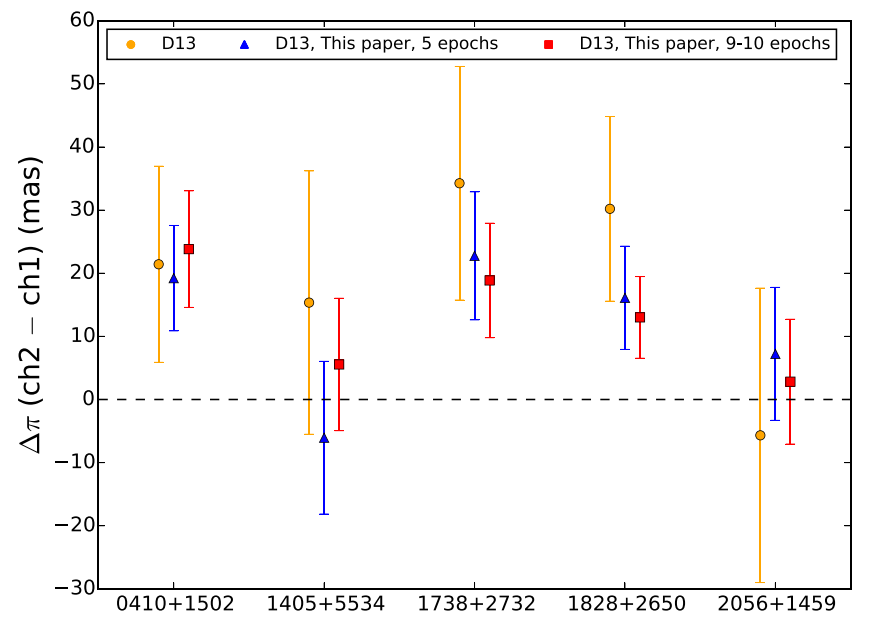

Figure 10. Comparison of parallaxes measured with [4.5] and [3.6], for targets overlapping the Dupuy \& Kraus (2013) data set. Parallax difference (mas) is plotted for each overlapping target. Data points have been offset to better show uncertainties. Yellow circles are the original measurements from Dupuy \& Kraus (2013). Blue triangles were measured by re-reducing the Dupuy \& Kraus (2013) data and using our own fitting analysis. Red squares were measured using the Dupuy \& Kraus (2013) data set with 9-10 additional epochs of [3.6] data included from the Spitzer archive and were analyzed using our fitting code.

and adding additional epochs, we were unable to fully account for the discrepancy, but the offset as a function of color also appears to only have a slight trend.

\subsubsection{Comparison to Leggett et al. 2017}

Leggett et al. (2017) and Luhman \& Esplin (2016) used Spitzer [4.5] data from our parallax program to measure astrometry for several of the objects in this paper. We note a $\sim 1$ - to 2- $\sigma$ offset that is largely systematic between their measurements and our own. They find larger parallaxes than we do, by $\sim 10-20$ mas for four out of six objects. It is unclear what is causing the difference between our parallax measurements.

\section{Discussion of $\mathbf{Y}$ dwarf Effective Temperatures}

\subsection{Not All Y Dwarfs are Created Equal}

Y0 dwarfs span several magnitudes in $M_{J}$, and nearly two in $W 2$, based on the near-infrared classification of Y0 dwarfs. As previously mentioned, we used $J-W 2$ as a proxy for temperature to separate populations in Figure 7. The color cuts show that the Y0 class spans $>4$ mag in $J-W 2$ and also overlaps the $J-W 2$ color space occupied by the Y1 and latertyped objects. These findings indicate that the classical nearinfrared spectral typing method of sorting $\mathrm{M}, \mathrm{L}$, and $\mathrm{T}$ dwarfs by their $J$ band spectral morphologies does not efficiently separate $\mathrm{Y}$ dwarfs by their respective temperatures. Y dwarfs, with $T_{\text {eff }} \lesssim 500 \mathrm{~K}$, emit only a small fraction of their light in the near-infrared and would be best-characterized based on their mid-infrared spectra. This was noted in the Y dwarf discovery paper, Cushing et al. (2011); however, until the launch of JWST, observers have little hope of obtaining high $\mathrm{S} / \mathrm{N}$ mid-infrared spectra of Y dwarfs- though some have tried (e.g., Skemer et al. 2016). The peak emission of a $\lesssim 500 \mathrm{~K}$ brown dwarf falls in the $\sim 3-10 \mu \mathrm{m}$ range, causing the $J$ band to lie on the Wien tail of the blackbody spectrum. Considering the above, we recommend that mid-infrared spectra (i.e., from 
JWST) be used to more fully characterize the physical properties of these extremely cold objects. Below we examine some of the more interesting targets in our sample.

\subsection{Notes on Specific YO Dwarfs}

WISE $0146+4234$ AB: This object has discrepant nearinfrared photometry in the literature due to its blended binary nature. For this reason, we have excluded it from our color cuts and plot it in gray in Figures 6 and 7.

WISE 0336-0143: This exhibits absolute magnitude and colors much more similar to $\mathrm{Y}$ dwarfs, than late-T dwarfs, as seen in Figures 6 and 7. We currently only have a limit on its near-infrared magnitudes, but our photometry agree with the later epoch of spectroscopy that this object is indeed a Y dwarf.

WISE 0734-7157: This particular dwarf is likely one of the warmest Y0's, based on its color and $M_{J}$. The best-fit temperature from Schneider et al. (2015) is $450 \mathrm{~K}$, and Leggett et al. (2017) estimate its $T_{\text {eff }}$ to be $435-465 \mathrm{~K}$.

WISE 1639-6847: This is the second coldest Y0, based on $J-W 2$ color. It is location in color-magnitude space is much more similar to the Y1 objects. Leggett et al. (2017) estimate its $T_{\text {eff }} \sim 360-390 \mathrm{~K}$, coinciding with our findings.

WISE 2209+2711: This is the faintest Y0 dwarf in every absolute magnitude band we measure. It is also the reddest in Y0 in $J-W 2$. From Schneider et al. (2015), the best fit model gives $T_{\text {eff }}=500-550 \mathrm{~K}, \quad \log g=4-4.5, \quad 0.2-1.5 \mathrm{Gyr}$ old. Leggett et al. (2017) estimate $T_{\text {eff }}=310-340 \mathrm{~K}$, which agrees better with our estimates that this object is colder than most Y0's. Even if we re-classify this as a Y1, this would still be the faintest and reddest $\mathrm{Y} 1$. This object is also the reddest $\mathrm{Y} 0$ in $J-H$ and $H-W 2$. It is mildly blue but not unusual in $Y-J$ (Schneider et al. 2015). If we use the $J-W 2$ versus Temperature plot from Schneider et al. (2015) to determine an effective temperature, this object should be only $\sim 300 \mathrm{~K}$. At such cold temperatures, the near-infrared flux is solely coming from the Wien tail. Our observations are thus not able to fully sample the peak of the Planck function, and thus a small shift in $T_{\text {eff }}$ can cause a significant change in absolute magnitudes and colors. This particular target would be excellent for follow-up with JWST spectroscopy and imaging.

\subsection{Notes on Specific Y0.5 Dwarfs}

WISE $0825+2805$ : This target is the third-reddest object in this sample in $J-W 2$ after WISE $1828+2650$ and WISE 2209 +2711 , likely indicating its extremely cold nature.

\subsection{Notes on Specific $\geqslant Y 1$ Dwarfs}

WISE 0350-5658 is the reddest in [3.6]-[4.5] in this sample, also the faintest in $M_{[3.6]}$ and $M_{\text {[4.5], and the faintest Y1 }}$ in $M_{J}$. It is likely extremely cold, probably matching the predicted $\sim 300 \mathrm{~K}$ from Schneider et al. (2015) and the 310-340 K from Leggett et al. (2017).

WISE $0535-7500$ is the brightest Y1-classified object and yet it was classified as $\geqslant Y 1$ in Kirkpatrick et al. (2012). WISE 0535 is located on the outskirts of the Large Magellanic Cloud and is in a highly crowded field that partially contaminated the $H S T$ spectrum. This object would also benefit from follow-up observations in the mid-infrared.

WISE $1828+2650$ is a known outlier that has thus far evaded a satisfactory explanation. Leggett et al. (2017) propose that the peculiar near- and mid-infrared colors could be due to an unseen or equal-mass binary, however there are a couple of problems with the binarity hypothesis. First, extreme redness cannot be explained with binarity. Based on evolutionary models, extremely cold Y dwarfs effectively cannot be young, and so a protoplanetary or debris disk makes an unlikely culprit for the enhanced [3.6] and [4.5]. Second, the amount by which this object is over-luminous is at least one mag (depending on the band) and the maximum over-brightness observed from an equal-mass binary is $0.75 \mathrm{mag}$.

\subsection{Other Findings}

\section{Summary and Final Remarks}

We present updated distance measurements for 22 late-T and Y dwarfs, measured from Spitzer/IRAC [4.5] data obtained over baselines of $\sim 2-7$ years. We also present the discovery of one new Y dwarf and five new late-T dwarfs, based on spectra from Keck/NIRSPEC. With these distances, we probe the physical properties of $\mathrm{Y}$ dwarfs, and find that the $\mathrm{Y}$ dwarf spectral classifications are likely not ordering objects in a temperature-sensitive sequence. JWST mid-infrared spectra will probe the peak of the spectral energy distributions of these ultracool dwarfs and provide a better understanding of their physical characteristics.

The discovery of an additional Y dwarf, presented in this paper, brings the current total known Y dwarfs to 26. It has long been recognized that brown dwarfs cannot account for dark matter, and rather make up a fraction of the number of celestial objects compared to stars. However, it is likely that our sample of Y dwarfs within $\sim 10-20 \mathrm{pc}$ is incomplete. Their extremely cold nature makes them difficult to detect in proper motion surveys. A dedicated 3-5 $\mu \mathrm{m}$ all sky survey with a smaller pixel scale than WISE would likely find a handful more. CatWISE ${ }^{25}$ is an upcoming survey that will use a re-processing of the AllWISE data to find fainter proper motion sources, including potential Y dwarfs. SPHEREx, ${ }^{26}$ a proposed NASA MIDEX mission, would conduct an all-sky spectral survey across $1-5 \mu \mathrm{m}$ and would also be likely to find more nearby $\mathrm{T}$ and $\mathrm{Y}$ dwarfs.

These objects are ideal for follow-up with JWST as we try to better understand star formation at the lowest masses and probe atmospheric conditions at the coldest temperatures. It may eventually be possible to spectroscopically differentiate between field brown dwarfs that have cooled to $\mathrm{Y}$ dwarf temperatures from the lowest-mass, Jupiter-sized exoplanets that have been ejected from their host system. Differing formation mechanisms predict different metallicity contents, but determining a metallicity will require $3-10 \mu \mathrm{m}$ spectroscopy with JWST.

This work is based in part on observations made with the Spitzer Space Telescope, which is operated by the Jet Propulsion Laboratory, California Institute of Technology under a contract with NASA. The authors wish to recognize and acknowledge the very significant cultural role and reverence that the summit of Mauna Kea has always had within the indigenous Hawaiian community. We are most fortunate to have the opportunity to conduct observations from this mountain. This publication makes

\footnotetext{
25 https://github.com/catwise

26 http://spherex.caltech.edu/index.html
} 
use of data products from WISE, which is a joint project of the University of California, Los Angeles, and the Jet Propulsion Laboratory/California Institute of Technology, funded by the National Aeronautics and Space Administration. This work has made use of data from the European Space Agency (ESA) mission Gaia (http://www.cosmos.esa.int/gaia), processed by the Gaia Data Processing and Analysis Consortium (DPAC, http://www.cosmos.esa.int/web/gaia/dpac/consortium). Funding for the DPAC has been provided by national institutions, in particular the institutions participating in the Gaia Multilateral Agreement. This research has made use of the NASA/ IPAC Infrared Science Archive, which is operated by the Jet Propulsion Laboratory, California Institute of Technology, under contract with the National Aeronautics and Space Administration. This work is based in part on observations made with the Hale Telescope at Palomar Observatory, which is operated by the California Institute of Technology.

R.L.S' research was supported by the 2015 Henri Chrétien International Research Grant administered by the American Astronomical Society.

E.C.M. thanks Dr. Gregory Mace for useful discussions, feedback, and mentoring.

Facilities: Spitzer(IRAC), Keck:II(NIRSPEC), Palomar (WIRC), IRSA.

Software: REDSPEC (http://www2.keck.hawaii.edu/inst/ nirspec/redspec.html); Astropy (Astropy Collaboration et al. 2013; The Astropy Collaboration et al. 2018); MOPEX (http:// irsa.ipac.caltech.edu/data/SPITZER/docs/dataanalysistools/tools /mopex/); Scipy (http://www.scipy.org/); Numpy (Oliphant 2006); Matplotlib (Hunter 2007).

Note added in proof. We also note that in Bedin \& Fontanive (2018), they measured a parallax for WISE J154151.65-225024.9 to be $169 \pm$ 2 mas, which is consistent with our own value of $167.1 \pm 4.2$ mas.

\section{ORCID iDs}

Emily C. Martin (1) https://orcid.org/0000-0002-0618-5128 J. Davy Kirkpatrick 나 https://orcid.org/0000-0003-4269-260X Jacqueline K. Faherty 나 https://orcid.org/0000-0001-6251-0573 Michael C. Cushing (i) https://orcid.org/0000-0001-7780-3352 Adam C. Schneider (i) https://orcid.org/0000-0002-6294-5937 Edward L. Wright (i) https://orcid.org/0000-0001-5058-1593 Patrick Lowrance (ib https://orcid.org/0000-0001-8014-0270 James Ingalls (1) https://orcid.org/0000-0003-4714-1364 C. G. Tinney (i) https://orcid.org/0000-0002-7595-0970 Sarah E. Logsdon (ii) https://orcid.org/0000-0002-9632-9382 Jérémy Lebreton iㅣ https://orcid.org/0000-0003-1476-5963

\section{References}

Astropy Collaboration, Robitaille, T. P., Tollerud, E. J., et al. 2013, A\&A, 558, A33

Baraffe, I., Chabrier, G., Barman, T. S., Allard, F., \& Hauschildt, P. H. 2003 , A\&A, 402, 701

Bate, M. R. 2005, MNRAS, 363, 363

Bedin, L. R., \& Fontanive, C. 2018, MNRAS, submitted

Beichman, C., Gelino, C. R., Kirkpatrick, J. D., et al. 2014, ApJ, 783, 68

Burgasser, A. J. 2004, ApJS, 155, 191

Burrows, A., Hubbard, W. B., Lunine, J. I., \& Liebert, J. 2001, RvMP, 73, 719

Burrows, A., Marley, M., Hubbard, W. B., et al. 1997, ApJ, 491, 856
Burrows, A., Sudarsky, D., \& Lunine, J. I. 2003, ApJ, 596, 587

Carey, S. J., Surace, J. A., Glaccum, W. J., et al. 2010, Proc. SPIE, 7731, $77310 \mathrm{~N}$

Cushing, M. C., Hardegree-Ullman, K. K., Trucks, J. L., et al. 2016, ApJ, 823,152

Cushing, M. C., Kirkpatrick, J. D., Gelino, C. R., et al. 2011, ApJ, 743, 50

Cushing, M. C., Kirkpatrick, J. D., Gelino, C. R., et al. 2014, AJ, 147, 113

Dupuy, T. J., \& Kraus, A. L. 2013, Sci, 341, 1492

Dupuy, T. J., \& Liu, M. C. 2012, ApJS, 201, 19

Dupuy, T. J., Liu, M. C., \& Leggett, S. K. 2015, ApJ, 803, 102

Faherty, J. K., Riedel, A. R., Cruz, K. L., et al. 2016, ApJS, 225, 10

Filippazzo, J. C., Rice, E. L., Faherty, J., et al. 2015, ApJ, 810, 158

Gaia Collaboration, Brown, A. G. A., Vallenari, A., et al. 2016a, A\&A, 595, A2

Gaia Collaboration, Prusti, T., de Bruijne, J. H. J., et al. 2016b, A\&A, 595, A1

Green, R. M. 1985, Spherical Astronomy (Cambridge and New York: Cambridge Univ. Press)

Hunter, J. D. 2007, CSE, 9, 90

Ingalls, J. G., Krick, J. E., Carey, S. J., et al. 2012, Proc. SPIE, 8442, 84421Y Kirkpatrick, J. D., Cushing, M. C., Gelino, C. R., et al. 2011, ApJS, 197, 19

Kirkpatrick, J. D., Gelino, C. R., Cushing, M. C., et al. 2012, ApJ, 753, 156

Kirkpatrick, J. D., Martin, E. C., Smart, R. L., et al. 2018, ApJ, submitted

Kirkpatrick, J. D., Schneider, A., Fajardo-Acosta, S., et al. 2014, ApJ, 783, 122

Leggett, S. K., Cushing, M. C., Hardegree-Ullman, K. K., et al. 2016, ApJ, 830,141

Leggett, S. K., Morley, C. V., Marley, M. S., et al. 2013, ApJ, 763, 130

Leggett, S. K., Morley, C. V., Marley, M. S., \& Saumon, D. 2015, ApJ, 799, 37

Leggett, S. K., Tremblin, P., Esplin, T. L., Luhman, K. L., \& Morley, C. V. 2017, ApJ, 842, 118

Line, M. R., Marley, M. S., Liu, M. C., et al. 2017, ApJ, 848, 83

Line, M. R., Teske, J., Burningham, B., Fortney, J. J., \& Marley, M. S. 2015, ApJ, 807, 183

Low, C., \& Lynden-Bell, D. 1976, MNRAS, 176, 367

Lowrance, P. J., Carey, S. J., Ingalls, J. G., et al. 2014, Proc. SPIE, 9143, 914358

Luhman, K. L. 2013, ApJL, 767, L1

Luhman, K. L. 2014, ApJL, 786, L18

Luhman, K. L., Burgasser, A. J., \& Bochanski, J. J. 2011, ApJL, 730, L9

Luhman, K. L., \& Esplin, T. L. 2016, AJ, 152, 78

Mace, G. N., Kirkpatrick, J. D., Cushing, M. C., et al. 2013a, ApJS, 205, 6

Mace, G. N., Kirkpatrick, J. D., Cushing, M. C., et al. 2013b, ApJ, 777, 36

McCallon, H. L., Fowler, J. W., Laher, R. R., Masci, F. J., \& Moshir, M. 2007, PASP, 119, 1308

McLean, I. S., Becklin, E. E., Bendiksen, O., et al. 1998, Proc. SPIE, 3354, 566

McLean, I. S., McGovern, M. R., Burgasser, A. J., et al. 2003, ApJ, 596, 561

Metchev, S. A., Heinze, A., Apai, D., et al. 2015, ApJ, 799, 154

Morley, C. V., Fortney, J. J., Marley, M. S., et al. 2012, ApJ, 756, 172

Morley, C. V., Marley, M. S., Fortney, J. J., et al. 2014, ApJ, 787, 78

Oliphant, T. E. 2006, Guide to Numpy (Cambridge, MA: MIT), http://web. mit.edu/dvp/Public/numpybook.pdf

Padoan, P., Nordlund, A., Kritsuk, A. G., Norman, M. L., \& Li, P. S. 2007, ApJ, 661, 972

Saumon, D., \& Marley, M. S. 2008, ApJ, 689, 1327

Saumon, D., Marley, M. S., Abel, M., Frommhold, L., \& Freedman, R. S. 2012, ApJ, 750, 74

Schneider, A. C., Cushing, M. C., Kirkpatrick, J. D., et al. 2015, ApJ, 804, 92

Skemer, A. J., Morley, C. V., Allers, K. N., et al. 2016, ApJL, 826, L17

Skrutskie, M. F., Cutri, R. M., Stiening, R., et al. 2006, AJ, 131, 1163

Smart, R. L., Apai, D., Kirkpatrick, J. D., et al. 2017, MNRAS, 468, 3764

Smart, R. L., Ioannidis, G., Jones, H. R. A., Bucciarelli, B., \& Lattanzi, M. G. 2010, A\&A, 514, A84

Smart, W. M., \& Green, R. M. 1977, in Textbook on Spherical Astronomy, ed. W. M. Smart \& R. M. Green (Cambridge: Cambridge Univ. Press), 446

The Astropy Collaboration, Price-Whelan, A. M., Sipőcz, B. M., et al. 2018, AJ, 156, 123

Tinney, C. G., Faherty, J. K., Kirkpatrick, J. D., et al. 2012, ApJ, 759, 60

Tinney, C. G., Faherty, J. K., Kirkpatrick, J. D., et al. 2014, ApJ, 796, 39

Wilson, J. C., Eikenberry, S. S., Henderson, C. P., et al. 2003, Proc. SPIE, 4841,451

Winters, J. G., Sevrinsky, R. A., Jao, W.-C., et al. 2017, AJ, 153, 14

Wright, E. L., Eisenhardt, P. R. M., Mainzer, A. K., et al. 2010, AJ, 140, 1868

Zuckerman, B., \& Song, I. 2009, A\&A, 493, 1149 